\title{
Arsenic Speciation Analysis by Ion Chromatography - A Critical Review of Principles and Applications
}

\author{
Adrian A. Ammann \\ EAWAG, Swiss Federal Institute of Aquatic Science and Technology, Dübendorf, Switzerland \\ E-mail: adrian.ammann@eawag.ch \\ Received August 1, 2010; revised January 6 2011; accepted January 10, 2011
}

\begin{abstract}
Multiple acute and chronic toxicity of arsenic species and its mobilisation from geological deposits into ground and drinking water resources are one of the greatest threats to human health. Arsenic speciation analysis, mostly done by liquid chromatography, is a challenging task which requires an intense high quality work with respect to extraction, preservation, separation, detection and validation. A growing number of As-species and low regulatory limits $(10 \mu \mathrm{g} / \mathrm{L})$ may require more than one speciation method preferably performed by species specific procedures and detectors. Beside As-fractionation for special application there are many selective speciation methods based on high performance separation techniques like capillary electrophoreses, gas and liquid chromatography. Both, fractionation and speciation methods are reviewed. However, the focus is on scopes and limits of ion chromatographic separations, the most frequently used methods. Based on IC-principles the methods applied are critically discussed and recommendations given which should result in more robust and reliable As-speciation.
\end{abstract}

Keywords: Review, Arsenic Speciation, Ion Chromatography

\section{Introduction}

Accumulating evidence on multiple toxicity aspects [1] including mutagenic, teratogenic and general genotoxic $[2,3]$ and neurotoxicity [4] effects of several arsenic species curbed down regulatory limits to $10 \mu \mathrm{g} / \mathrm{L} \mathrm{As}$ (WHO, US-EPA) in drinking water. Considering chronic toxicological effects in combination with As-mobilisation from geological deposits into ground water [5], an essential source of drinking water, As is likely to pose one of the greatest threat to human health worldwide. Since years this is reality for up to 100 million people in India [6], Bangladesh [7,8], Vietnam [9,10], Cambodia [11] and other places on the world [1]. Fears have been expressed [12] that this is only the visible tip of the iceberg. These intensified As-problems initiated many investigations to reassess the mobilisation, transformation and toxicity of even low concentrated As-species.

Among oxyanion-forming elements, As shows a unique mobilisation [5] over the $\mathrm{pH}$-range of natural waters under both oxidising and reducing conditions. Inorganic redox species arsenite (AsIII) and arsenate (AsV) are far the most abundant and the most toxic species in environmental waters. In biologically mediated transforma- tions these species are converted into numerous organic As-species [13,14]. All As-related problems like toxicity, adsorption and transport, biogeochemical cycling and treatment of drinking water depend on As speciation.

For several reasons As speciation remains a challenging task which requires a lot of high quality speciation work: the toxicity of many As-species is not jet elucidated and often a single separation procedure is not reliable since most toxic species can be interfered by As-species of much lower toxicity [15]. Arsenic has a unique rich chemistry [16] with a huge number of organic As-species [17] reflecting the capability of this element to adapt to almost any condition. On the other hand, investigations addressing a large number of species are inevitably forced to reduce costs. However, cost reduction can become so dominant that it restricts the number of questions which can be answered, or worse, narrowing it to what can be achieved by the lowest cost procedure which is unable to answer important open questions. Another difficulty is that the separation procedure has to be adapted to the species preservation or vice versa. But, what is the best preservation during storage and separation? It depends on the matrix and the As-species present. However, if the latter are not com- 
pletely known one finds itself in a real speciation dilemma.

Previous reviews provide a helpful orientation by emphasizing different aspects in As-speciation. An over view on recent reviews can be found in [18]. Francesconi et al. [19] gave short synopsis and characterisations of all the different methods in a broad (450 references) and comprehensive coverage. The nomenclature given there is followed in this review and listed in Table 1 together with other abbreviations. The chemistry of As-species in the aquatic environment was extensively reviewed [20] just recently. B'Hymer et al. [21] focused on the role of HPLC coupled to ICP MS. Analytical methods for inorganic As have been reviewed by Hung et al. [22] and

Table 1. Names and abbreviations of chemicals ${ }^{\mathrm{a}}$.

\begin{tabular}{|c|c|c|}
\hline Abbrev. & Names & Formula \\
\hline \multicolumn{3}{|l|}{ Arsenicals } \\
\hline AsIII & Arsenite (arsenous acid) & $\mathrm{As}(\mathrm{OH})_{3}$ \\
\hline $\mathrm{AsV}$ & Arsenate (arsenic acid) & $\mathrm{AsO}(\mathrm{OH})_{3}$ \\
\hline $\mathrm{AB}$ & Arsenobetaine & $\left(\mathrm{CH}_{3}\right)_{3} \mathrm{As}^{+} \mathrm{CH}_{2} \mathrm{COO}^{-}$ \\
\hline $\mathrm{AC}$ & Arsenocholine & $\left(\mathrm{CH}_{3}\right)_{3} \mathrm{As}^{+}\left(\mathrm{CH}_{2}\right)_{2} \mathrm{OH}$ \\
\hline DMA & Dimethylarsinic acid & $\left(\mathrm{CH}_{3}\right)_{2} \mathrm{AsO}(\mathrm{OH})$ \\
\hline MMA & Monomethylarsonic acid & $\mathrm{CH}_{3} \mathrm{AsO}(\mathrm{OH})_{2}$ \\
\hline TETRA & Tetramethylarsonium ion & $\left(\mathrm{CH}_{3}\right)_{4} \mathrm{As}^{+}$ \\
\hline TMAO & Trimethylarsineoxide & $\left(\mathrm{CH}_{3}\right)_{3} \mathrm{AsO}$ \\
\hline p-ASA & p-Aminobenzenearsonic acid & p- $\mathrm{NH}_{2} \mathrm{C}_{6} \mathrm{H}_{4} \mathrm{OAs}(\mathrm{OH})_{2}$ \\
\hline p-PSA & 4-Hydroxybenzenearsonic a. & $\mathrm{p}-\mathrm{OHC}_{6} \mathrm{H}_{4} \mathrm{OAs}(\mathrm{OH})_{2}$ \\
\hline Rox & 4-HO-3-nitrophenylarsonic a. & $\mathrm{OHNO}_{2} \mathrm{C}_{6} \mathrm{H}_{3} \mathrm{OAs}(\mathrm{OH})_{2}$ \\
\hline \multicolumn{3}{|l|}{ Other } \\
\hline $\mathrm{AcO}$ & Acetate & $\mathrm{CH}_{3} \mathrm{COOH}$ \\
\hline BDSA & Benzene-1,2-disulfonic acid & $\mathrm{C}_{6} \mathrm{H}_{4}\left(\mathrm{SO}_{3}\right)_{2}{ }^{2-}$ \\
\hline Cit & Citric acid & $\left(\mathrm{CH}_{2} \mathrm{COOH}\right)_{2} \mathrm{COHCOOH}$ \\
\hline DL & Detection limit & \\
\hline EDTA & Ethylenediaminetetraacetate & $\left(\left(\left(\mathrm{OOCCH}_{2}\right)_{2} \mathrm{~N}\right)_{2}\left(\mathrm{CH}_{2}\right)_{2}\right.$ \\
\hline HSA & Hexanesulfonic acid & $\mathrm{CH}_{3}\left(\mathrm{CH}_{2}\right)_{5} \mathrm{SO}_{3} \mathrm{H}$ \\
\hline PS-DVB & Polystyrene-divinylbezene & \\
\hline SDS & Sodiumdodecylsulfate & $\mathrm{CH}_{3}\left(\mathrm{CH}_{2}\right)_{11} \mathrm{OSO}_{3} \mathrm{H}$ \\
\hline Tart & Tartrate & $\left(\mathrm{CH}_{2}\right)_{2}\left(\mathrm{COO}^{-}\right)_{2}$ \\
\hline TBA & Tertrabutylammonium & $\left(\mathrm{C}_{4} \mathrm{H}_{9}\right)_{4} \mathrm{~N}^{+}$ \\
\hline TRIS & $\begin{array}{l}\text { Tris (hydroxymethyl) } \\
\text { amino-methan }\end{array}$ & $\left(\mathrm{HOCH}_{2}\right)_{3} \mathrm{CNH}_{2}$ \\
\hline
\end{tabular}

a) The nomenclature follows the recommendations given by Francesconi et al. [19]. voltammetric methods for the same purpose just recently by Mays et al. [23].

For a toxicity assessment, ionic As-oxo-species are far more decisive than others and hence ion chromatography (IC) the most often applied method. In this review advantages and shortcomings of specific IC procedures are critically discussed, rather than completely covering in details all the numerous As-speciation methods.

\section{Methods for As Speciation}

Arsenic speciation analysis can be categorized according to the speciation definition given by IUPAC [24] which is based on selectivity and specificity. Methods which inherently cannot differentiate among chemical species are named fractionation and those with a higher or tuneable resolution are called speciation methods. Following this definition, distinctions like AsIII and AsV, inorganic and organic or smaller and larger size belongs to fractionation since each of this categories consists of several chemical species which are indistinguishable by such methods. So chromatographic separations, except size exclusion (SEC), are considered speciation methods since they can be tuned or used in combinations [25] to separate essentially all chemical species.

A successful speciation requires not only an accurate species determination but also to be in an optimal accordance with the sample treatment (extraction, preservation). Because many speciation methods are based on IC-principles, particular attention is paid to IC-methods and their applications.

\subsection{Arsenic Fractionations}

\subsubsection{Inorganic AsIII/AsV Fractionation}

The fact that the two most toxic As-compounds are the inorganic AsIII and AsV justify this fractionation for samples influenced by pure geochemical processes (e.g. groundwater) where no or very minor fraction of organic arsenicals occur. The hydride generation, oxidation state selective fractionation, makes use of the ease As-compounds form hydrides under different conditions: AsIII reacts at slightly acidic $\mathrm{pH}$ (6) with borohydrides whereas $\mathrm{AsV}$-arsenicals require $\mathrm{pH} 1$ or a pre-reduction, e.g. by thiols (cysteine [26,27], thioglycolic acid [28]). However, organic arsenicals can require harsh oxidation conditions $\left(\mathrm{HNO}_{3} / \mathrm{HClO}_{4} / \mathrm{H}_{2} \mathrm{SO}_{4}\right.$ at $\left.300^{\circ} \mathrm{C}\right)$ for decomposing, especially $\mathrm{AC}$ and $\mathrm{AB}$ are the most recalcitrant [29]. Once formed, As-hydrides are volatile and easily separable from the matrix by gas liquid separators or gas diffusion [30]. After this step, they can be diverted directly to a detector or cryo-trapped, pre-concentrated and selectively evaporated in the sequence of their boiling points [31]. 
In-situ AsIII/AsV fractionation can also be obtained by very sensitive electrochemical methods $(20 \mathrm{ng} / \mathrm{L}$ $[32,33])$ which are continuously developed [23]. By exclusively measuring AsIII at natural sample $\mathrm{pH}$ 7-8 and $\mathrm{AsIII}+\mathrm{AsV}$ in an acidified (pH 1) sample aliquot, $\mathrm{AsV}$ is assigned to the difference of the two AsIII measurement [32].

\subsubsection{Extraction and Preservation Procedures}

The time between sampling and analysis often requires a preservation which has not only to be reliable but also compatible with the subsequent speciation method, which is often a difficult task. Species extraction from difficult matrices (soil, sediments, food) can alter the As-speciation and pose a problem for separation procedures.

Most extraction procedures have to be considered as fractionation because of the inherent difficulty to extract completely all species [34-37] by a single procedure. The widespread use of one single extraction method only inspired others to define a new class of "hidden species" $[38,39]$. Even in case of a hypothetical $100 \%$ recovery of each species, inherent procedural species instabilities can cause a shift in species ratio which is difficult to prevent (e.g. instabilities of the oxidation states $[14,40]$, or the thio vs oxo-form [41-43]). A control might be possible for a limited set of As-species as they occur in certified reference materials $[44,45]$, despite total As only is certified in the material.

A variety of extraction procedure have been developed and optimized for several matrices: terrestrial plants [46-48], algae and aquatic plants [49], soils [50-52], food [52] and microwave-assisted extraction from soil [53] and vegetables $[54,55]$. Solvent extraction (SE) are now investigated with a high enrichment factor (115) [56], ultrasound assisted [57,58] and microwave assisted SE [59], solid phase micro-extraction [60-64].

For the stabilization of As-species low temperatures were sufficient, e.g. freezing (liquid $\mathrm{N}_{2}$ ) for clinical samples [65] and $-16^{\circ}$ for algae extracts [66]. Moreover, diverse additives have been proposed: methanol [52], mineral acids like $\mathrm{HCl}$ [67-69] and phosphoric acid [52], phosphoric acid in combination with cooling $\left(6^{\circ}\right)$ [70,71], chelators like EDTA [72-74] against metal precipitation. Some of these preservation procedures have been compared $[40,69]$ using the same set of samples. Low temperatures in combination with preservatives which account for matrix particularities are most effective.

\subsubsection{Specialized Fractionation Procedures}

In recent years, there has been some progress in isolating special As-fractions like bio-available, volatile or size fractions, exclusively defined by the procedure applied.
A bio-available fraction has been obtained extracting seafood continuously by synthetic body-fluids (saliva, stomach- and gastric juice) [75] and by an in vivo assay [76]. Capture of volatile As-species is indispensable for a total As-balance in a dumpsite [77] or in biogas [78].

Biological samples are often fractionated on a size exclusion column to isolate as much as possible As-species in one run from the complex matrix. This can serve as a cleaning step for further chromatographic speciation [79]. SEC was useful in detecting thioarsenicals as As-phytochelatins from plants [80] and methylated thio-arsenicals in urine [81].

\subsection{Sensors and Field Tests}

In a large area with a high number of sampling sites it gets impractical to collect and transfer samples to a laboratory. It is more convenient to perform analysis or tests on site in the field and evaluate critical samples only in the laboratory. However, the recommended limit for As $(10 \mathrm{ppb}(\mu \mathrm{g} / \mathrm{L}))$ is a very tough hurdle for a sensor or field test which usually operate with compromised detection limits, accuracy and precision in favour of low cost portable instrumentation [82] or test kits [83,84]. If hundreds of volunteers are required, simple and fast test kits are indispensible which can be easily handled by non-scientific staff. Such a screening fulfils a different purpose that is to provide approximate concentrations allowing a sample classification and splitting (below, around and above a limit) for a reduced workload. Detailed requirements and the chemistry applied by field test were discussed and reviewed [85,86]. Biologicalbased assays have been developed and are reviewed in [87]. Microelectronic sensing of the biological response to arsenicals [88] has a not yet exploited potential.

\subsection{As-Speciation Using IC Methods}

\subsubsection{Detectors in As-Speciation}

The method sensitivity has not only a decisive influence on which samples (concentrations) can be analyzed or which method has to be chosen to analyze envisaged species in given samples (concentrations), but the performance and the complexity of the method too depend on the sensitivity. E.g. lower sensitivity detectors require sample clean up procedures and pre-concentrations steps which in turn increase the labour load and the possibility of speciation alterations. In chromatography, the detector sensitivity is linked to the chromatographic performance as a more sensitive detector allows sample dilution or larger dilution factors. More diluted samples can show less severe matrix interference, thus enabling a more robust procedure. 
Sensitivity and providing species specific information are the two main abilities detectors meet As-speciation demands. Detectors which are sensitive to only a small number of species require that other species must be chemically transformed in high recovery prior to detection which can cause problems. Bulkiness and costs are other important properties. The smaller, less sensitive and less expensive detectors like UV, conductivity and chemiluminescence [30] are not As-specific and not sensitive enough, whereas the more demanding (cost and space) are more sensitive and deliver species specific data. Among the specific detectors there are atomic fluorescence (AFS) and electrochemical instruments which can be applied to a few As-species only. Volatile hydrides are required for detection by AFS [58,89-91] and only inorganic AsIII [92,93], or AsIII/AsV [32] by electrochemistry. Element-specific detectors like atomic absorption (AAS) and optical emission (OES) traditionally used to determine total element concentrations provided a first generation of universal As-species detectors.

However, sensitivity requirements in As-speciation can bee roughly 1-2 order of magnitude more demanding compared to total element determinations since the element is distributed on several species and many of them have to be separated and detected individually. Element mass spectrometers (MS) extend the detection limit (DL) noticeably. Simple quadrupole ICP MS are the most widely used detectors in general trace element speciation analysis [94-96] as well as in arsenic speciation [21] since they are versatile and the most sensitive instruments [97]. The same is valid for a high resolution (HR) ICP MS except that its sensitivity is at least 10 times better [98]. The particular advantages of plasma source MS have been pointed out in [21,97,99].

Organic MS, detecting molecules and molecule fragments, responded to research and validation needs for more information and proof of the species structure [97]. The advantages and the limitations of various detectors and their couplings to different separation methods are discussed in $[100,101]$. Considerable evidence in reliable structural assignment was obtained by divers molecule and fragment ionisation MS techniques $[100,102]$ and by $\mathrm{x}$-ray absorption [103] that gives information on Asbonding in the solid state [104-107] which can eliminate artifacts generating extraction steps.

\subsubsection{Selective Separation Methods}

A sensitive As-specific detector coupled to a sufficiently selective separation method is the heart-piece in Asspeciation [108-110]. In most cases a traditional high performance separation technique such as gas chromatography (GC), capillary zone electrophoresis (CE), several liquid chromatographic (LC) methods like ion ex- change chromatography (IEC) and ion pair chromatography (IPC) on reversed phase HPLC columns are linked to diverse detectors according to the analytical task.

Volatile arsenicals found under natural conditions were separated highly efficient by GC. Arsenolipids were determined in fish oil [111]. Several mixed arsenosulfur compounds which were produced by intestinal microorganisms [112] were analyzed. Typically, volatile arsenicals are produced in derivatization steps like hydride generation $[113,114]$ and methyl thioglycolate derivatives were extracted into hexane and determined by GC atomic emission [115]. With respect to the growing number of volatile arsenicals, GC remains an important separation method as reviewed in [116]. However, many naturally occurring arsenicals are not volatile and not stabile at the temperature required to keep them in the gas phase. For these compounds liquid separation methods like CE and LC are better suited.

High separation efficiencies made $\mathrm{CE}$ an attractive method [97]. However, its low amount of analyte mass applied in combination with low sensitivity detectors provides insufficient DL. For the most common As-species, DL of 5-17 $\mu \mathrm{g} / \mathrm{L}$ were reached [117], detecting with a high sensitivity UV-cell and high sample volume stacking. In situ heteropolyacid formation with molybdate and UV-detection gave similar DL [118]. Without sensitivity enhancement in UV-detection, DL between 0.1 and $1.2 \mathrm{mg} / \mathrm{L}$ have been reported [119] in aqueous soil extracts. UV detection was $10^{3}-10^{4}$ times less sensitive compared with ESI MS [120] and with ICP MS [121]. CE coupled to more sensitive detectors requires special interfaces and attention to some particular issues $[122,123]$. With ICP MS, DL of $0.04 \mu \mathrm{g} / \mathrm{L}$ have been reported [124]. Comparing CE and IEC both coupled to the same HR ICP MS gave 100 times higher DL with CE [125].

HPLC and Ion Chromatography. The most often encountered As-species cover the whole range of molecule polarities, e.g. anions (AsV, MMA, DMA), cations (AC, TMA) and, depending on the $\mathrm{pH}$, neutral molecules (AB, AsIII) [126]. The diverse polarities, the growing number and the different types of As-compounds are a permanent challenge to IC. The method should be robust in routine speciation analysis, provide lowest DL, and separate as many as possible of diverse As-species. Strategies for arsenic speciation analysis have been presented by Larsen [126] and Feldmann et al. [13].

As the most toxic As-species are ionic, the overwhelming part in As-speciation is done by IC. IC-methods were previously reviewed, but IC principles and their role within the whole context of As-speciation (species stability and preservation, on column stability, 
detector compatibility and variable selectivity) has not been critically discussed, making it difficult for nonexperts to gain a comprehensive understanding. This review is intended to better clarify the role of IC in As-speciation and to highlight improved procedures. The different types of IC methods [127], e.g. anion exchange (AEX), cation exchange (CEX), ion exclusion (IEC), ion pair chromatography (IPC) and combinations there of are potentially suitable for As-speciation and are discussed below.

The affinity of an analyte ion towards an ion exchanger depends on the charge density and the polarizability of the two opposite charges. For a given exchanger material, the ion density is given by the number of exchange sites per material mass (capacity) and the polarizability is similar among columns functionalized by the same ionic groups. So far, $\mathrm{R}_{4} \mathrm{~N}^{+}, \mathrm{SO}_{3}{ }^{-}, \mathrm{RCOO}^{-}$were most often used as ion exchanging groups [127,128]. The analytes charge density and polarizability depends on the molecule size and the charge which is often controlled by a proton association-dissociation equilibrium. The $\mathrm{pK}_{\mathrm{a}}$ of arsenicals are spread over a large range, but many of them are $\mathrm{pK}_{\mathrm{a}}>8$ [129]. Hence, their negative charge depends on the $\mathrm{pH}$ and the same is valid for protonable or deprotonable column exchange sites. All these variables are controlled by the eluent-pH which becomes the master variable. Unfortunately, the $\mathrm{pH}$ of the frequently used eluents (see below) cannot be freely adjusted to the As-species $\mathrm{pK}_{\mathrm{a}}$ to tune their retention behaviour and improve the selectivity of the method [130]. The $\mathrm{pH}$ is rather dictated by the column capacity that requires an equivalent eluent concentration to reach the eluent strength that can elute species. With such eluents, there is no other choice than to perform the separation at a fixed value or, in case of acids and bases, even at an extreme $\mathrm{pH}(<3$ or $>9)$ where many As-compounds are not stabile. Such extreme eluent-pH practically excludes silicabased columns, so separations are usually done with synthetic columns. On organic polymers, ion exchange can be combined with hydrophobic interaction on the column core material, aiding in retention of neutral Asspecies.

Adjusting the analyte charge density by the eluent $\mathrm{pH}$ can be best realized by AEX [126]. In the beginning of ion exchange development it was found [131] that AEX separated common As-species whereas CEX did not retain the two most toxic and most common species, AsIII and $\mathrm{AsV}$, but eluting them together in the front. This has been confirmed in many follow-up comparisons. A recently developed CEX gradient separation $(0-20 \mathrm{mM}$ ${ }^{+} \mathrm{NH}_{4}, \mathrm{pH}=2.5$ [77]) on a PRP-X200 column improved the separation somewhat, but again confirmed the prob- lems by eluting AsIII, MMA, AsV and $\mathrm{Cl}^{-}$(interfering as ${ }^{40} \mathrm{Ar}^{35} \mathrm{Cl}^{+}$on ${ }^{75} \mathrm{As}^{+}$-detection by ICP MS [132]) within 3 min in the front and co-eluting AC with TAMO at 15 min. A more promising CEX, just recently published [133], uses acidified $(\mathrm{HCOOH})$ acetonitrile.

Therefore AEX is recommended $[13,134]$ as the primary separation and, if required, CEX as a secondary option to separate the less toxic organic cationic arsenicals not resolved on an anion exchanger. This can be achieved by a dual mode method, which consists on one hand of an additionally applied CEX procedure (as two columns two procedures [13] or as two columns in series in one procedure $[135,136])$. On the other hand, a mixed mode ion exchanger (mostly AS7 column,) can be used, containing both, anionic and cationic exchange sites [127]. In many cases, AEX, single or mixed mode, sufficiently separates a restricted number of cationic arsenicals, making a separate CEX-run obsolete.

Ion Pair Chromatography [137]. In IPC a simple reversed phase column is dynamically coated by a lipophilic counter ion in the eluent. While this is an attractive access to IC without purchasing additional expensive covalently functionalised columns, problems arise by the dynamic exchange capacity which depends on the sample matrix. Results obtained by this technique until 2002 were reviewed in [134] and an overview is given in Table 2. The dynamic in-situ coating in AEX was compared to permanent bound exchanger sites. The latter method [138] gave better results because of the higher and more stabile exchange capacity. The dynamic coating can easily be disrupted by matrix components [139], e.g. salts and surface active organics of unknown concentrations. It was also demonstrated that the concentration of the ion pair reagent is very critical, a slight excess can reduce the selectivity substantially [140] and a calculation assisted method optimization found no ion pairing effect at all [59]. Nevertheless, it has been shown that a dynamic ion pair coating withstanding the coat disrupting ability of the sample matrix and an accurately developed method can result in robust and unique separation conditions [141]. Ion pairing reagents can also strongly increase the separation efficiency of AEX (see below).

Anion Exchange Chromatography. AEX separations are done almost exclusively on higher capacity, highly hydrophobic synthetic columns from several manufacturers (Table 3-5) and with eluents which severely restrict the eluent-pH. Accordingly, three types of restrictions can be distinguished for the following discussion: 1) a strong acidic $\mathrm{HNO}_{3}$-eluent at two isocratic steps $(\mathrm{pH}<3)$ mostly on a mixed-mode anion-cation exchanger AS7 (Table 3), 2) Strongly basic eluents like carbonate or hydroxide $(\mathrm{pH}$ $>8.5$ ) on diverse columns (Table 4) and 3) phosphate 
Table 2. Ion pair chromatographic separations.

\begin{tabular}{|c|c|c|c|c|c|c|c|}
\hline $\begin{array}{l}\text { IP reagent } \\
(\mathrm{mM})\end{array}$ & $\begin{array}{c}\text { Eluent } \\
(\mathrm{pH})\end{array}$ & Modifier & Matrix & $\begin{array}{l}\text { As species }^{\mathbf{a}} \\
\left(\mathrm{t}_{\mathrm{R}}, \mathrm{min}\right)\end{array}$ & $\begin{array}{l}\text { Detector } \\
(\mathrm{DL}, \mu \mathrm{g} / \mathrm{L})\end{array}$ & Comment & Ref. \\
\hline \multicolumn{8}{|l|}{ anion pairing } \\
\hline TBAOH (5) & $\mathrm{H}_{2} \mathrm{O}(6.0)$ & $4 \% \mathrm{MeOH}$ & & $\begin{array}{c}\text { AB, AsIII, DMA, MMA } \\
\text { AsV (12.5) }\end{array}$ & ICP MS (3) & matrix interf. & [139] \\
\hline $\mathrm{TBAPO}_{4}(2.5)$ & $\mathrm{H}_{2} \mathrm{O}(5.2)$ & & & $\begin{array}{l}\text { AsIII, AB, AC, DMA, } \\
\text { MMA AsV (8.0) }\end{array}$ & HG AAS (100) & $\begin{array}{l}\mathrm{AB}, \mathrm{AC} \\
\text { coelute }\end{array}$ & {$[157]$} \\
\hline $\mathrm{TBAPO}_{4}(10)$ & $\mathrm{PO}_{4} 20 \mathrm{mM}(6.0)$ & & & $\begin{array}{c}\text { AsIII, DMA, MMA AsV } \\
(9.0)\end{array}$ & HG AAS (100) & & {$[172]$} \\
\hline TBAOH (5) & $\mathrm{H}_{2} \mathrm{O}(6.2)$ & & $\begin{array}{l}\text { wine, kelp } \\
\quad \text { extr. }\end{array}$ & $\begin{array}{c}\text { AsIII DMA, MMA AsV } \\
\text { (1.7), pPSA, } \\
\text { As-sugars }\end{array}$ & ICP MS (0.7) & $\begin{array}{l}\text { narrow bore } \\
\text { HPLC }\end{array}$ & {$[140]$} \\
\hline TBAOH (13) & $\mathrm{H}_{2} \mathrm{O}(5.8)$ & $1.3 \% \mathrm{MeOH}$ & urine & $\begin{array}{c}\text { AB, AsIII, DMA, MMA } \\
\text { AsV (6.4) }\end{array}$ & $\operatorname{ICP} \mathrm{MS}(0.2)$ & & {$[173]$} \\
\hline $\mathrm{TBAPO}_{4}(5)$ & $\mathrm{H}_{2} \mathrm{O}(5.9)$ & & & $\begin{array}{c}\text { AsIII, DMA, MMA AsV } \\
\text { (8), pASA }\end{array}$ & ICP MS (4) & nano HPLC & {$[174]$} \\
\hline TBAOH (5) & $\mathrm{PO}_{4} \mathrm{SO}_{4}(6.0)$ & & $\begin{array}{l}\text { urine, plant } \\
\text { extract, river } \\
\text { water }\end{array}$ & $\begin{array}{l}\text { AsIII, DMA, MMA AsV } \\
\text { (6.2) } 4 \text { Se-comp. }\end{array}$ & ICP MS (0.02) & $\begin{array}{l}\text { simult. As and } \\
\text { Se - speciation }\end{array}$ & [141] \\
\hline \multicolumn{8}{|l|}{ cation pairing } \\
\hline SDS (10) & $\mathrm{H}_{2} \mathrm{O}(2.5)$ & $\begin{array}{l}5 \% \mathrm{MeOH} 5 \\
2.5 \% \mathrm{AcOH}\end{array}$ & fish tissue & $\begin{array}{c}\text { AB, AsIII, DMA, MMA } \\
\text { AsV (12.5) }\end{array}$ & ICP MS (3) & & [139] \\
\hline HSA (10) & 40 mM Cit (2.3) & $\begin{array}{l}2-12 \% \mathrm{MeOH} \\
\text { gradient }\end{array}$ & apple extr. & $\begin{array}{l}\text { AsV (1.8) AsIII, MMA, } \\
\text { DMA, AB, AC (24) }\end{array}$ & ICP MS & $\begin{array}{l}\text { AsIII, MMA } \\
\text { coelute }\end{array}$ & {$[175]$} \\
\hline
\end{tabular}

a) The elution sequence is given.

Table 3. Anion exchange with eluents restricted to low pH $\left(\mathrm{HNO}_{3}\right)$.

\begin{tabular}{|c|c|c|c|c|c|c|c|}
\hline $\begin{array}{l}\text { Column } \\
\text { (capacity) }\end{array}$ & $\begin{array}{c}\text { Eluent }^{\mathbf{b}} \\
\mathrm{HNO}_{3} \text { steps }\end{array}$ & Modifier & Matrix & $\begin{array}{c}\text { As species }^{c} \\
\left(t_{R}\right)\end{array}$ & $\begin{array}{c}\text { Detector } \\
(\mathrm{DL}, \mu \mathrm{g} / \mathrm{L})\end{array}$ & Comment & Ref. \\
\hline AS7 (125) & $0.5,50$ & $0.05 \mathrm{BDSA}$ & & AsIII, DMA, AsV, AB, AC, & ICP MS (0.5) & & {$[142][143][176]$} \\
\hline AS7 (125) & $0.5,50$ & $0.05 \mathrm{BDSA}$ & plant extract & & & & {$[177]$} \\
\hline AS7 (125) & $0.5,50$ & 0.05 BDSA & $\begin{array}{l}\text { oyster, rice, } \\
\text { algae }\end{array}$ & 17 arsenicals & $\begin{array}{c}\text { ICP MS } \\
(0.01-0.05)\end{array}$ & $\mathrm{AB} \& \mathrm{Cl}^{-}$coelute & {$[145][146]$} \\
\hline AS7 (125) & $2.5,50$ & non & poultry waste & $\begin{array}{c}\text { AsIII, MMA, AsV, DMA, } \\
\text { ROX }\end{array}$ & $\begin{array}{l}\text { ICP MS } \\
(0.02)\end{array}$ & & {$[144]$} \\
\hline AS7 (125) & $0.5,50$ & $1 \% \mathrm{MeOH}$ & seafood extr. & $\begin{array}{c}\text { AsIII, MMA, DMA, AsV } \\
\text { (6), AB, AC }\end{array}$ & $\begin{array}{c}\text { ICP MS } \\
(0.01-0.04)\end{array}$ & $\begin{array}{l}\text { calc.assisted } \\
\text { se.optimization }\end{array}$ & {$[59]$} \\
\hline AS4 (25) & $0.4,50$ & non & algae extract & $\begin{array}{l}\text { AsIII, MMA, DMA, AsV } \\
\text { (6), AB, AC }\end{array}$ & $\begin{array}{l}\text { ICP MS } \\
(0.03-1.5)\end{array}$ & $\begin{array}{l}\text { better without } \\
\text { modif. }\end{array}$ & {$[178]$} \\
\hline
\end{tabular}

a) Manufacturer of AS7 and AS4: Dionex. b) Step concentrations in mM. c) Elution sequence is given.

eluents of various composition and $\mathrm{pH}$ on different columns (Table 5). Separations based on these procedures have been compared [72] to a field speciation method using more than 100 surface waters, ground waters, and acid mine drainage samples. Alternatively, the advantages of non $\mathrm{pH}$ restricting eluents (see Table 6) are discussed. In some work, organic anions were used as eluent ions which produce a high carbon load to ICP detectors. In low concentrations and as polycarboxylates they play a role as ionic strength modifier.

Strong acidic eluents on the mixed mode exchanger
AS7 were often used (compare Table 4) to separate anionic, cationic and neutral As-species [142]. The high hydrophobic nature of the exchange sites provided additional selectivity in conjunction with an ion pair reagent [143] and methanol [144]. The strongly acidic BDSA, which is anionic at low $\mathrm{pH}\left(\mathrm{pK}_{\mathrm{a} 1}<1\right)$, was so far the most beneficial one. It interacts better with the positively charged As-centre than with the positive charges on the column, forming more negatively charged ion pairs with neutral or positive arsenicals [143]. Best separation is achieved by eluting at two isocratic nitric acid concentra- 
Table 4. Anion exchange with eluents restricted to high $\mathrm{pH}\left(\mathrm{HO}^{-}, \mathrm{CO}_{3}{ }^{2-}\right)$.

\begin{tabular}{|c|c|c|c|c|c|c|c|}
\hline $\begin{array}{l}\text { Columnn } \\
\text { (capacity) }\end{array}$ & $\begin{array}{l}\text { Eluent mM } \\
\quad(\mathrm{pH})\end{array}$ & Modifier & Matrix & $\begin{array}{l}\text { As species }^{\mathbf{b}} \\
\left(\mathrm{t}_{\mathrm{R}}, \mathrm{min}\right)\end{array}$ & $\begin{array}{l}\text { Detector } \\
(\mathrm{DL}, \mu \mathrm{g} / \mathrm{L})\end{array}$ & Comment & Ref. \\
\hline $\begin{array}{l}\text { ICPak MS }^{1} \\
\text { IC AN2 }^{2}\end{array}$ & $\begin{array}{c}\mathrm{HCO}_{3}^{-} 25(9.25) \\
\mathrm{CO}_{3}{ }^{-2} 100(10.25)\end{array}$ & - & soil extracts & $\begin{array}{c}\text { AB, AC, TMA, DMA, AsIII, } \\
\text { MMA, AsV }\end{array}$ & ICP MS (3) & $\begin{array}{l}\mathrm{AB}, \mathrm{AC}, \mathrm{TMA} \\
\text { coelute }\end{array}$ & [138] \\
\hline ICPakA HC $^{1}$ & $\mathrm{CO}_{3}^{2-} 2.5(10.25)$ & - & & $\begin{array}{l}\text { AB, DMA, AsIII, MMA, AsV, } \\
\text { AC }\end{array}$ & ICP MS (0.1) & $\begin{array}{l}\text { dual column } \\
\text { system }\end{array}$ & [135] \\
\hline IC AN2 & $\mathrm{CO}_{3}^{2-} 50(10.3)$ & - & groundwater & $\begin{array}{c}\mathrm{AB}, \mathrm{DMA}, \mathrm{AsIII}, \mathrm{MMA}, \mathrm{AsV} \\
(8)\end{array}$ & ICP MS (0.2) & $60^{\circ}$ & {$[126]$} \\
\hline $\operatorname{AS} 11(56)^{3}$ & $\mathrm{NaOH} 30$ & $1 \% \mathrm{MeOH}$ & sediment & AsIII, AsV (3.5) & HG AAS (1) & & {$[152]$} \\
\hline AS11 (56) & $\mathrm{NaOH} 0-40$ & - & urine & $\begin{array}{c}\mathrm{AB}, \mathrm{DMA}, \mathrm{AsIII}, \mathrm{MMA}, \mathrm{AsV} \\
(9.1)\end{array}$ & $\begin{array}{l}\text { ICP MS } \\
(0.1-0.8)\end{array}$ & $\begin{array}{c}\text { AB, MMAIII } \\
\text { and DMAIII } \\
\text { not sep. }\end{array}$ & [155] \\
\hline $\operatorname{AS} 14(80)^{3}$ & $\begin{array}{c}\mathrm{NH}_{4} \mathrm{HCO}_{3} 2(8.2) \\
\text { Tart } 2 \text { or } 45\end{array}$ & - & & AsIII, DMA, MMA, AsV & ICP MS (0.5) & Se-species & {$[156]$} \\
\hline $\operatorname{AS} 9 H C(237)^{3}$ & $\mathrm{Na}_{2} \mathrm{CO}_{3}$ 5-70 (12) & - & $\begin{array}{l}\text { food stuff } \\
\text { extracts }\end{array}$ & AB, DMA, AsIII, MMA, AsV & ICP MS (0.3) & $\begin{array}{l}\text { AsIII is } \\
\text { oxidised }\end{array}$ & [149] \\
\hline $\operatorname{AS} 16(174)^{3}$ & $\begin{array}{l}\mathrm{NaOH} 30,50 \\
\quad(12.7)\end{array}$ & $1 \% \mathrm{MeOH}$ & poultry waste & $\begin{array}{l}\text { DMA, AsIII, MMA, p-ASA, } \\
\text { AsV (6.6), Rox(9.2) }\end{array}$ & ICP MS (0.02) & & [144] \\
\hline $\operatorname{AS} 16(174)^{3}$ & $\mathrm{NaOH} 1.5-55$ & - & groundwaters & $\operatorname{AsV}(26)$ & suppr. conduct. & $\begin{array}{c}\text { other } \\
\text { oxyanions }\end{array}$ & [153] \\
\hline $\begin{array}{l}\text { PRP X100 } \\
\quad(190)\end{array}$ & $\begin{array}{c}\mathrm{NH}_{4} \mathrm{HCO}_{3} 15-50 \\
(8.5)\end{array}$ & - & $\begin{array}{l}\text { lobster tissue } \\
\text { extr. }\end{array}$ & $\begin{array}{l}\text { AC, AB, AsIII, DMA, } \\
\text { MMA(17), AsV (25) }\end{array}$ & ICP MS & & {$[154]$} \\
\hline AS9HC $(237)^{3}$ & $\mathrm{Na}_{2} \mathrm{CO}_{3} 3,70(12)$ & & soil water & AB, DMA, AsIII, MMA, AsV & $\begin{array}{l}\text { HR ICP MS } \\
\quad(0.01)\end{array}$ & spilt $1: 10$ & [125] \\
\hline AS7 $(125)^{3}$ & $\mathrm{NH}_{4} \mathrm{CO}_{3} 0-25(10)$ & & algae extr. & DMA, AsIII, MMA, AsV (30) & ICP MS (0.01) & & {$[179]$} \\
\hline $\operatorname{As} 11(56)^{3}$ & $\mathrm{NaOH} 0-40$ (12.6) & & urine & $\begin{array}{c}\mathrm{AB}, \mathrm{DMA}, \mathrm{AsIII}, \mathrm{MMA}, \mathrm{AsV} \\
(7.2)\end{array}$ & ICP MS (0.02) & & [155] \\
\hline PRP X100 & $\mathrm{NH}_{4} \mathrm{CO}_{3} 20(9.0)$ & & molluscs & thio As-sugars & & & {$[180]$} \\
\hline PRP X100 & $\mathrm{NH}_{4} \mathrm{CO}_{3} 20(10)$ & & & 50 As-species & $\begin{array}{c}\text { ESI SRM } \\
\text { MSMS }(0.02)\end{array}$ & & {$[17]$} \\
\hline PRP X100 & $\mathrm{NH}_{4} \mathrm{CO}_{3} 10-50(9)$ & $2 \% \mathrm{MeOH}$ & urine, fish & $\begin{array}{c}\mathrm{AB}, \mathrm{AsIII}, \mathrm{DMA}, \mathrm{MMA}, \mathrm{AsV} \\
(7)\end{array}$ & $\begin{array}{l}\text { DRC ICP MS } \\
(0.01)\end{array}$ & Se-compounds & [181] \\
\hline
\end{tabular}

a) Manufacturer (1-4): 1 Waters (polymetacrylat), 2 Merck, 3 Dionex (PS-DVB), 4 Hamilton (PS-DVB). b) Elution sequence is given. ESI SRM MSMS: electrospray ionisation selected reaction monitoring tandem MS. DRC: dynamic reaction cell.

tion $(0.5$ and at $50 \mathrm{mmol} / \mathrm{L}$ (pH 3.4 and 1.3 resp.), but a gradient is not successful. The procedure $[145,146]$ was developed to a high resolution IC method. A concentration gradient of the ion pairing reagent, however, resulted in irreproducible retention times $\left(t_{R}\right)$ of all analytes [143] and methanol in the sample can cause different response for As-species [146]. Surprisingly, independent work $[59,147]$ reported that the BDSA-concentration has no influence on the separation. With respect to metal precipitation on the column, such an acidic eluent was considered preferable because it keeps metals in solution and continuously removes metals from the column. However, on column deposition of matrix components and loss of chromatographic performance after a few sample injections have been reported [143] for $\mathrm{HNO}_{3}$ eluents as well. The aggressive and oxidising nitric acid significantly reduces the lifetime of organic polymeric column material. While the procedure is perfectly compatible with the sample introduction and the plasma of an ICP instrument, it is not compatible with sample preservation procedure and on column stabilities of sensitive As-compounds. Oxidation of AsIII to AsV in nitric acid preserved samples [40] and large differences in species concentrations compared to other IC methods [144] have been observed. The oxidation was found to be catalysed by metals [148] and mediated too by light [69].

High $\mathrm{pH}$ eluents substantially increase the dissociation of protonated As-species and increase their affinity for the anion exchanger. The advantages of this type of AEX were discussed by Larson [126]. Under oxic basic conditions oxidation of AsIII to $\mathrm{AsV}$ is fast and was reported to occur during chromatography $[40,144,149,150]$. This method seems to be suited for $\mathrm{NaOH}$ extracted soil samples, to perform the separation without adjusting the sample $\mathrm{pH}$. Beside $\mathrm{K}_{2} \mathrm{SO}_{4}(10 \mathrm{mmol} / \mathrm{L}, \mathrm{pH}$ 10.2) [151], hydroxide and carbonate containing eluents have widely been used on a variety of polymeric anion exchanger columns (see Table 4). The higher the column capacity 
Table 5. Anion exchange with eluents restricted to medium $\mathrm{pH}$ values $\left(\mathrm{PO}_{4}\right.$, org. acids).

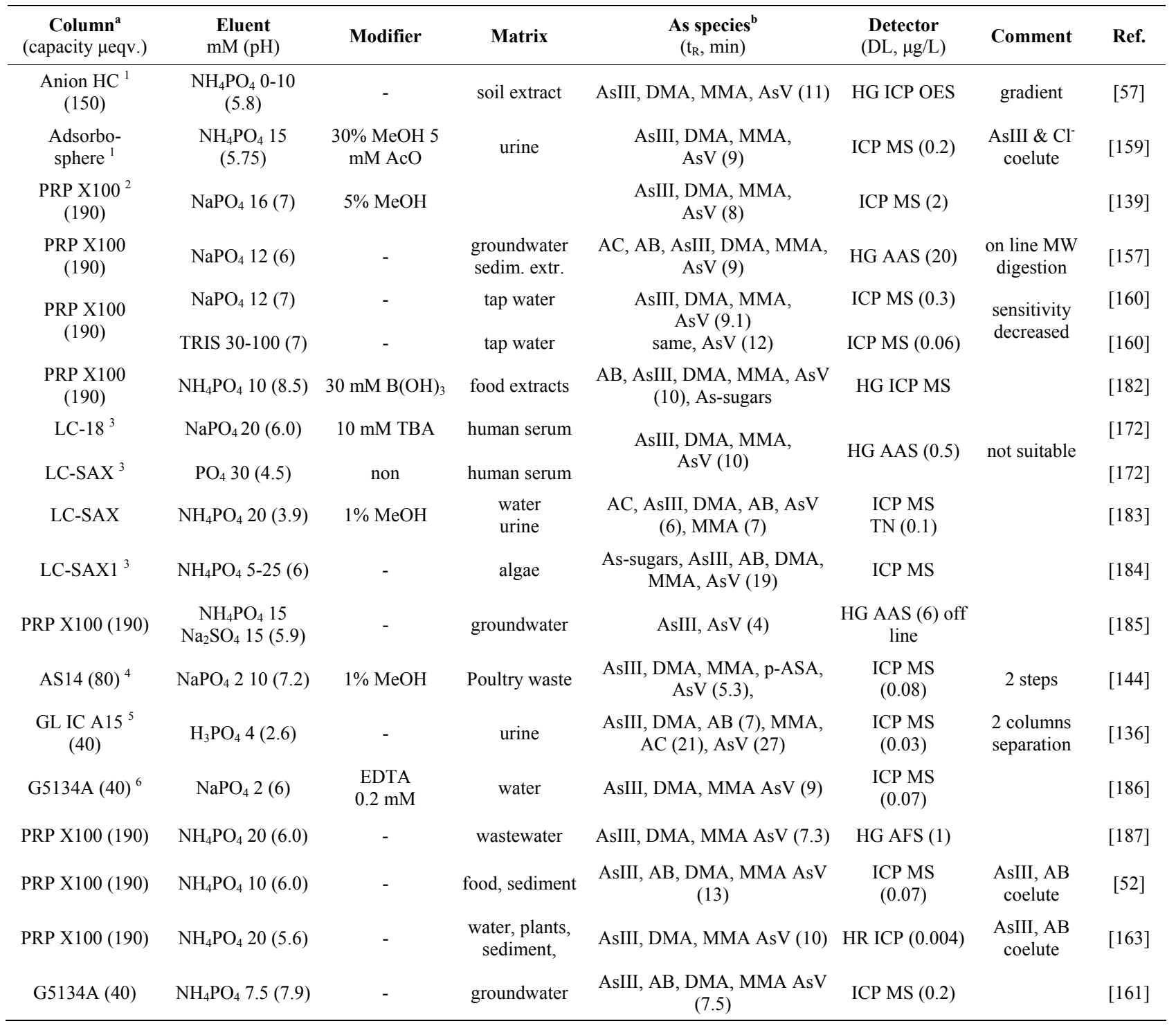

a) Manufacturer (1-6): 1 Alltech (Si-C18), 2 Hamilton, 3 Supelco (LC-SAX (Si-N ${ }^{+}(B u t)$, LC-SAX1 $\left(\mathrm{Si}_{-} \mathrm{N}^{+}\left(\mathrm{CH}_{3}\right)_{3}\right), 4$ Dionex, 5 Agilent, 6 Hitachi. HG: Hydride Generation. MW: microwave. TN: thermospray nebulization.

is, the higher the eluent concentration (isocratic or gradient ramp) has to be. On a low capacity AS11 short $t_{R}$ were obtained applying $30 \mathrm{mmol} / \mathrm{L}$ hydroxide [152] whereas on a AS16 column [144,153] up to $55 \mathrm{mmol} / \mathrm{L}$ were required. Short $t_{R}(<4 \mathrm{~min})$ for $\mathrm{AsV}$ were reported [138] with high isocratic carbonate concentrations, 25 $\mathrm{mmol} / \mathrm{L}$ and $\mathrm{pH}=9.25$ on IC-PAK-HS or $100 \mathrm{mmol} / \mathrm{L}$ and $\mathrm{pH}=10.25$ on IC-AN2. Carbonate up to $70 \mathrm{mmol} / \mathrm{L}$ ( $\mathrm{pH} 12$ ) had to be applied on a AS9HC column to separate As-species within 5 minutes [40]. The advantage of a gradient $(15-30 \mathrm{mmol} / \mathrm{L})$ vs. isocratic $(30 \mathrm{mmol} / \mathrm{L}) \mathrm{se}-$ paration was demonstrated [154] using $\mathrm{NH}_{4} \mathrm{HCO}_{3} \mathrm{pH}=$ 8.5 on a PRP-X100 column. The gradient provided almost baseline separation of the cationic AC and the neu- tral AB. However, the low eluting power of the $\mathrm{HCO}_{3}$ anion required over 26 minutes to elute $\mathrm{AsV}$ from this column. A hydroxide gradient $(0-40 \mathrm{mM} \mathrm{NaOH})$ on AS11 was applied to separate inorganic and organic AsIII and AsV in urine samples [155].

A low carbonate concentration $(2.5 \mathrm{mmol} / \mathrm{L}, \mathrm{pH}=9.3)$ was applicable on IC-PAK-A-HC $(150 \times 4.6 \mathrm{~mm})$ serially connected to a cation exchange column [135]. Carbonate and hydroxide were used in combination, e.g (5 $\mathrm{mmol} / \mathrm{L}, 20 \mathrm{mmol} / \mathrm{L}$ resp.) on $\mathrm{AS} 12$ column [71] and (5 $\mathrm{mmol} / \mathrm{L}, 40 \mathrm{mmol} / \mathrm{L}$ resp.) on AS4-SC [142]. In order to lower the $\mathrm{pH}$ but increase the ionic strength, inorganic eluent anions have been combined with organic anions, e.g. $\mathrm{NH}_{4} \mathrm{HCO}_{3}(2 \mathrm{mmol} / \mathrm{L})$ with tartrate $(2$ and $40 \mathrm{mmol} / \mathrm{L}$, 
Table 6. Anion and cation exchange with an eluent of a large pH-variability $\left(\mathrm{NH}_{4} \mathrm{NO}_{3}\right)$.

\begin{tabular}{|c|c|c|c|c|c|c|c|}
\hline $\begin{array}{l}\text { Column } \\
\text { (capacity) }\end{array}$ & $\begin{array}{c}\text { Eluent }^{\mathbf{b}} \\
\mathrm{NH}_{4} \mathrm{NO}_{3} \\
\end{array}$ & pH & Matrix & $\begin{array}{l}\text { As species } \\
\left(t_{R}, \min \right)\end{array}$ & $\begin{array}{l}\text { Detector } \\
(\mathrm{DL}, \mu \mathrm{g} / \mathrm{L})\end{array}$ & Comment & Ref. \\
\hline \multicolumn{8}{|l|}{$A E X$} \\
\hline PRP-100X & 20,60 & 8.7 & surf. water & $\begin{array}{l}\text { AsIII, DMA, MMA, AsV (7.1), } \\
\text { SeIV, SeVI, CrVI }\end{array}$ & $\begin{array}{l}\text { ICP MS } \\
(0.05)\end{array}$ & multielemental det. & [169] \\
\hline PRP-100X & 4,60 & 8.7 & $\begin{array}{l}\text { earthworm } \\
\text { extracts }\end{array}$ & $\begin{array}{c}\mathrm{AB}, \mathrm{AsIII}, \mathrm{DMA}, \mathrm{MMA}, \mathrm{AsV} \\
(9)\end{array}$ & $\begin{array}{l}\text { ICP MS } \\
(0.3-1)\end{array}$ & & [188] \\
\hline PRP-100X & 5,80 & 8.3 & env. waters & $\begin{array}{l}\text { AsIII, DMA, MMA, AsV (7.1), } \\
\text { SeIV, SeVI, }\end{array}$ & $\begin{array}{l}\text { ICP MS } \\
(0.03-0.04)\end{array}$ & micro IC-system & [171] \\
\hline AS11 (14) & $0.5-70$ gradient & 8.3 & groundwater & AB, AsIII, DMA, MMA, AsV & $\begin{array}{l}\text { HR ICP MS } \\
(0.005-0.01\end{array}$ & narrow bore system & {$[130]$} \\
\hline Methacrylate $^{1}$ & 15 & 3 & test water & $\begin{array}{l}\text { AsV (7), MMA, DMA, AsIII } \\
\text { (10.7), AB }\end{array}$ & $\begin{array}{l}\text { ICP MS } \\
(0.02-0.4)\end{array}$ & $\begin{array}{l}\text { AsV \& } \mathrm{Cl}^{-} \text {coelute } \\
\text { multimode exch. }\end{array}$ & {$[170]$} \\
\hline \multicolumn{8}{|l|}{ CEX } \\
\hline PRP-200X ${ }^{2}$ & $0-20$ gradient & 2.5 & landfill leachate & $\begin{array}{l}\text { AsIII, MMA, AsV (4), DMA, } \\
\text { AB, TETRA, AC (15), TMAO }\end{array}$ & $\begin{array}{l}\text { ICP MS } \\
(0.01-0.03)\end{array}$ & & [77] \\
\hline
\end{tabular}

a) Manufacturer as in Tables 2-5, 1 Micromass nano IC capillary, 2 Hamilton. b) Step or gradient concentrations in mM. c) Elution sequence is given.

$\mathrm{pH}=8.2)$ [156]. Separations at a high $\mathrm{pH}$ can suffer from metals $(\mathrm{Mg}, \mathrm{Ca}, \mathrm{Al}, \mathrm{Mn}, \mathrm{Fe}, \mathrm{Cu}$, etc) precipitating as hydroxides providing fresh adsorbing surfaces for Asspecies.

Phosphate as an eluent anion was used since a long time because of its particular advantages, but there are also some shortcomings which have to be considered. Compared to other eluents, phosphate is a real buffer able to alter on column the $\mathrm{pH}$ of the injected sample to the eluent $\mathrm{pH}$. As an AsV-analogue, it plays an indispensable role in displacing AsV from strong adsorbing sites and providing optimal recoveries in chromatography [130] or in extraction procedures [57]. Higher capacity anion exchangers (compare Table 5) required higher eluent $\mathrm{pH}$ and/or high phosphate concentrations to compete for arsenicals and elute $\mathrm{AsV}$ in a reasonable $t_{R}$. On the other hand, phosphorus and sulphur in the plasma produce polymeric depositions on the cones and inside of an ICP instrument. Therefore, procedures to reduce the P-load to the instrument have been developed. As-species were often transferred into volatile As-hydride and separated from the eluent in a post column reaction [72]. Organic As-species, however, react sluggishly at room temperature and need a particular fast on line digestion procedure [157] to reach a comparable mass transfer from a column effluent into the gas phase. A newly developed electrochemical hydride generation [158] might be an alternative in-line digestion for coupling to a detector. For a direct inlet into the ICP MS at $1 \mathrm{~mL} / \mathrm{min}$, phosphate concentration should clearly be lower than 15 $\mathrm{mmol} / \mathrm{L}$ since at this concentration a rapid damage of expensive cones [159] and a drop in sensitivity due to clogging [160] was reported. Recently it has been shown that ammonium instead of Na-salts in the plasma pro- duce less deposits [161]. Eluent splitting before the instrument inlet was used too in order to reduce the P-load. E.g., arsenate was eluted with $20 \mathrm{mmol} / \mathrm{L}$ phosphate $(\mathrm{pH}$ $5.6, \mathrm{t}_{\mathrm{R}}=10 \mathrm{~min}$ ) from a PRP-X100 column [162] and the eluent was split before HR ICP MS detection [163]. In cases where an eluent splitting is not an option, lower phosphate concentrations are mandatory. On the PRPX100 column $12 \mathrm{mmol} / \mathrm{L}$ [157] and $10 \mathrm{mmol} / \mathrm{L}$ [52] phosphate have been applied which eluted AsV in 12 minutes and compounds in biological samples have been identified which strongly bind to the column and to AsIII producing artefacts in As-species ratios [65]. In combination with poly-anionic EDTA $(1 \mathrm{mmol} / \mathrm{L}), 2.6 \mathrm{mmol} / \mathrm{L}$ phosphate eluted $\mathrm{AsV}$ in 12 minutes as well and was splitless introduced into a HR ICP MS [164]. Just recently, a phosphate gradient $(0-10 \mathrm{mmol} / \mathrm{L}, \mathrm{pH}=5.8)$ has been applied on a shorter $(4.6 \times 100)$, high capacity column ( 150 requiv.), eluting $\mathrm{AsV}$ in 11 min [57].

Phosphate has less $\mathrm{pH}$-restrictions than acids or bases and can be applied within a middle pH-range (3-9). In this range the charge of the phosphate eluent anion varies (between -1 and -2) depending on the degree of deprotonation $\left(\mathrm{pK}_{\mathrm{a} 1}=2.1, \mathrm{pK}_{\mathrm{a} 2}=7.2, \mathrm{pK}_{\mathrm{a} 3}=12.6\right)$. Hence, approaching $\mathrm{pH} 7$, phosphate is much more ionized which drastically increases its eluent strength. This was used to selectively increase the $t_{R}$ of DMA $\left(\mathrm{pK}_{\mathrm{a}}=6.1\right)$ while the $t_{R}$ of other species remained [161]. Further deprotonation at higher $\mathrm{pH}$, however, counteracts the selectivity among other As-species since the increased negative charge on phosphate shortens $t_{R}$ of arsenicals $[157,161]$. This makes the separation of the most toxic AsIII from the non toxic $\mathrm{AB}$ impossible [52] as the two are separated only at a higher eluent $\mathrm{pH}$ (e.g. 10.7 [157] or 8.2 [164]). The problem was addressed by combining phosphate with 
other eluting anions which do not change its eluting strength with $\mathrm{pH}$ above seven, like EDTA [164] or $\mathrm{SO}_{4}{ }^{-2}$ [141].

Organic eluent anions were used for special separations. A steep gradient of potassium hydrogenphthalate (0-100 $\mathrm{mM}, 0-5 \mathrm{~min})$ separated organic from inorganic arsenicals on a short column $(25 \mathrm{~mm})$ in less than three minutes [165] and AsIII/AsV were separated $(12.5 \mathrm{mM}$ malonate and $17.5 \mathrm{mM} \mathrm{AcO} \mathrm{pH} \mathrm{4.8)} \mathrm{on} \mathrm{another} \mathrm{short}$ column $(50 \mathrm{~mm})$ in less than 2 minutes [72,73]. Such short $t_{R}$ were also obtained on a micro column (ANX 1606AS, CETAC) with tartrate (5 mM, pH 8.5) separating AsIII and AsV [40,166]. A TRIS-buffer gradient (30$100 \mathrm{mM}, \mathrm{pH}$ 7) on a PRP-X100 column gave a better separation compared to phosphate [160]. Non preserved organic eluents promote rapid bacterial growth in the eluent [73] and on the column. However, organic carbon eluents are not often applied since the carbon introduced into the plasma modulates the As-ion formation by factors [167]. Unfortunately, organic and even inorganic carbon can vary the As-response [168] detected by ICP in an unexpected manner, particularly after chromatography.

Alternative eluents. Non buffer salts open up interesting alternatives. Mostly $\mathrm{NH}_{4} \mathrm{NO}_{3}$ (Table 6) has been used as fully $\mathrm{pH}$-flexible and outstanding plasma compatible eluent. A two step method $(20 \mathrm{mM}$ and $60 \mathrm{mM}$ $\mathrm{NH}_{4} \mathrm{NO}_{3}, \mathrm{pH}$ 8.7) demonstrated these unique properties in multi-elemental speciation [169]. This eluent was also chosen for a reliable detection in nano multimode-IC (15 $\left.\mathrm{mM} \mathrm{NH} \mathrm{NO}_{3}, \mathrm{pH} 3,[170]\right)$, micro-AEX (5 mM and 80 $\mathrm{mM} \mathrm{NH} \mathrm{NO}_{3}$-steps, $\left.\mathrm{pH} 8.3,[171]\right)$ and in narrow bore AEX (0.5-70 mM NH${ }_{4} \mathrm{NO}_{3}$-gradient, $\left.\mathrm{pH} 8.3,[130]\right)$. Up to now, this is the only eluent used to perform the separation around the sample $\mathrm{pH}$ or at any other freely selectable $\mathrm{pH}$ since its eluent strength does not depend on the $\mathrm{pH}$. Large $\mathrm{NH}_{4} \mathrm{NO}_{3}$-gradients can be applied [130, 171] and independently an eluent-pH can be selected according to the analyte protonation equilibrium for retention time adjustment and selectivity optimisation [130, $169,171]$. The same eluent was also applied in gradient cation exchange separation $(0-20 \mathrm{mM} \mathrm{pH} 2.5)$ of Asspecies [77].

\section{Conclusions}

Most As-species separation was done by anion exchange, so it is worth to look for possible optimizations of this important technique. In trace As-species analysis by AEX, the column capacity and the sample matrix has a dominant influence on separation conditions. Columns caring a lower exchange capacity and requiring lower eluent concentrations are seldom applied despite the of- ten deployed ICP MS is sensitive enough allowing for sample dilution. Separating diluted samples on low capacity columns reduces the eluent load to the plasma and the sample matrix load to the column as well, which would be the most simple and convenient way to a more robust chromatography.

Facing increasing evidence on As-species instabilities and artefacts in the matrix and during separation, the adaption of the eluent-pH close to the sample-pH is mandatory which is just contrary to what was extensively done in As-speciation so far. What so ever, extreme eluent $\mathrm{pH}$ cannot be used without a rigorous control of the species ratios over the whole analytical process. Moreover, in order to use a gradient for the separation of a larger number of As-species and to use the eluent-pH as an independent parameter to tune and enhance the AEX-selectivity, the eluent-pH should be completely independent from the eluent strength which is clearly not the case for acids, bases and most buffer salts, their $\mathrm{pK}_{\mathrm{a}}$ being order of magnitudes different from those of As-species. A non-buffer eluent salt, $\mathrm{NH}_{4} \mathrm{NO}_{3}$, that is known to fulfil all the requirements like best plasma compatibility, $\mathrm{pH}$-adjustable to any $\mathrm{pH}$ without affecting ionic strength and high purity has rarely been used so far. For plasma based detectors, eluent components containing carbon, phosphorus and sulphur are problematic since they require a lot of attention due to plasma incompatibilities causing sensitivity variations between injections and during a run (carbon).

These few important issues demonstrate that there is still a potential ahead for serious improvements in Asspecies analysis by AEX.

\section{Acknowledgements}

Thanks are due to Dr. Harald Hagendorfer (EMPA, Switzerland) for helpful discussions.

\section{References}

[1] B. K. Mandal and K. T. Suzuki, "Arsenic Round the World: A Review," Talanta, Vol. 58, No. 1, 2002, pp. 201-235. doi:10.1016/S0039-9140(02)00268-0

[2] A. M. Florea, E. Dopp, G. Obe and A. W. Rettenmeier, "Genotoxicity of Organometallic Species," In: A. V. Hirner and H. Emons, Eds., Organic Metal and Metalloid Species in the Environment, Springer, Berlin, 2004, pp. 205-215.

[3] A. Hartwig, T. Schwerdtle and I. Walter, "Current Aspects on the Genotoxicity of Arsenite and Its Methylated Metabolites: Oxidative Stress and Interaction with the Cellular Response to DNA Damage," In: A. V. Hirner and H. Emons, Eds., Organic Metal and Metalloid Species in the Environment, Springer, Berlin, 2004, pp. 
217-234.

[4] A. Vahidnia, G. B. Van der Voet and F. A. de Wolf, “Arsenic Neurotoxicity - A Review," Human \& Experimental Toxicology, Vol. 26, No. 10, 2007, pp. 823-832. doi:10.1177/0960327107084539

[5] P. L. Smedley and D. G. Kinniburgh, "A Review of the Source, Behaviour and Distribution of Arsenic in Natural Waters," Applied Geochemistry, Vol. 17, No. 5, 2002, pp. 517-568. doi:10.1016/S0883-2927(02)00018-5

[6] D. Chakraborti, B. Das, M. M. Rahman, U. K. Chowdhury, B. Biswas, A. B. Goswami, et al., "Status of Groundwater Arsenic Contamination in the State of West Bengal, India: A 20-Year Study Report," Molecular Nutrition \& Food Research, Vol. 53, No. 5, 2009, pp. 542-551. doi:10.1002/mnfr.200700517

[7] N. Nahar, "Causes and Distribution of Arsenic Contamination in Bangladesh: Evidence from the Literature," Water Policy, Vol. 11, No. 3, 2009, pp. 362-378. doi:10.2166/wp.2009.045

[8] J. A. Plant, D. G. Kinniburgh, P. L. Smedley, F. M. Fordyce and B. A. Klinck, "Arsenic and Selenium," In: S. B. Lollar, Ed., Environmental Geochemistry, Elsevier-Pergamon, Treatise on Geochemistry, Oxford, 2005, pp. 17-50.

[9] M. Berg, H. C. Tran, T. C. Nguyen, H. V. Pham, R. Schertenleib and W. Giger, "Arsenic Contamination of Groundwater and Drinking Water in Vietnam: A Human Health Threat," Environmental Science \& Technology, Vol. 35, 2001, pp. 2621-2626. doi:10.1021/es010027y

[10] J. Buschmann, M. Berg, C. Stengel, L. Winkel, M. L. Sampson, P. T. K. Trang, et al., "Contamination of Drinking Water Resources in the Mekong Delta Floodplains: Arsenic and Other Trace Metals Pose Serious Health Risks to Population," Environment International, Vol. 34, No. 6, 2008, pp. 756-764. doi:10.1016/j.envint.2007.12.025

[11] J. Buschmann, M. Berg, C. Stengel and M. L. Sampson, "Arsenic and Manganese Contamination of Drinking Water Resources in Cambodia: Coincidence of Risk Areas with Low Relief Topography," Environmental Science \& Technology, Vol. 41, No. 7, 2007, pp. 2146-2152. doi:10.1021/es062056k

[12] D. Chakraborti, M. M. Rahman, K. Paul, U. K. Chowdhury, M. K. Sengupta, D. Lodh, et al., "Arsenic Calamity in the Indian Subcontinent: What Lessons Have Been Learned?" Talanta, Vol. 58, No. 1, 2002, pp. 3-22. doi:10.1016/S0039-9140(02)00270-9

[13] J. Feldmann, S. Devalla, A. Raab and H. R. Hansen, "Analytical Strategies for Arsenic Speciation in Environmental and Biological Samples," In: A. V. Hirner and H. Emons, Eds., Oranic Metal and Metalloid Species in the Environment, Springer, Heidelberg, 2004, p. 41.

[14] W. Goessler and D. Kuehnelt, "Sample Preparation for Arsenic Speciation," In: Z. Mester and R. Sturgeon, Eds., Comprehensive Analytical Chemistry, Elsevier, Amsterdam, 2003, pp. 1027-1044.

[15] G. P. Miller, D. I. Norman and P. L. Frisch, "A Comment on Arsenic Species Separation Using Ion Exchange,"
Water Research, Vol. 34, No. 4, 2000, pp. 1397-1400. doi:10.1016/S0043-1354(99)00257-2

[16] K. A. Francesconi, "Toxic Metal Species and Food Regulations Making a Healthy Choice," Analyst, Vol. 132, No. 1, 2007, pp. 17-20. doi:10.1039/b610544k

[17] V. Nischwitz and S. A. Pergantis, "Optimisation of an HPLC Selected Reaction Monitoring Electrospray Tandem Mass Spectrometry Method for the Detection of 50 Arsenic Species," Journal of Analytical Atomic Spectrometry, Vol. 21, No. 11, 2006, pp. 1277-1286. doi: $10.1039 / \mathrm{b} 607535 \mathrm{e}$

[18] D. J. Butcher, "Environmental Applications of Arsenic Speciation Using Atomic Spectrometry Detection," Applied Spectroscopy Reviews, Vol. 42, No. 1, 2007, pp. 1-22. doi:10.1080/05704920600939398

[19] K. A. Francesconi and D. Kuehnelt, "Determination of Arsenic Species: A Critical Review of Methods and Applications, 2000-2003," Analyst, Vol. 129, No. 5, 2004, pp. 373-395. doi:10.1039/b401321m

[20] V. K. Sharma and M. Sohn, "Aquatic Arsenic: Toxicity, Speciation, Transformations, and Remediation," Environment International, Vol. 35, No. 4, 2009, pp. 743-759. doi:10.1016/j.envint.2009.01.005

[21] C. B'Hymer and J. A. Caruso, “Arsenic and Its Speciation Analysis Using High-Performance Liquid Chromatography and Inductively Coupled Plasma Mass Spectrometry," Journal of Chromatography A, Vol. 1045, No 1-2, 2004, pp. 1-13. doi:10.1016/j.chroma.2004.06.016

[22] D. Q. Hung, O. Nekrassova and R. G. Compton, "Analytical Methods for Inorganic Arsenic in Water: A Review," Talanta, Vol. 64, No. 2, 2004, pp. 269-277. doi:10.1016/j.talanta.2004.01.027

[23] D. E. Mays and A. Hussam, "Voltammetric Methods for Determination and Speciation of Inorganic Arsenic in the Environment - A Review," Analytica Chimica Acta, Vol. 646, No. 1-2, 2009, pp. 6-16. doi:10.1016/j.aca.2009.05.006

[24] D. M. Templeton, F. Ariese, R. Cornelis, L. G. Danielsson, H. Muntau, H. P. Van Leeuwen, et al., "Guidelines for Terms Related to Speciation and Fractionation of Elements. Definitions, Structural Aspects, and Methodological Approaches," Pure and Applied Chemistry, Vol. 72, No. 8, 2000, pp. 1453-1470. doi:10.1351/pac200072081453

[25] J. Szpunar and R. Lobinski, "Multidimensional Approaches in Biochemical Speciation Analysis," Analytical and Bioanalytical Chemistry, Vol. 373, No. 6, 2002, pp. 404-411. doi:10.1007/s00216-002-1282-2

[26] J. Frank, M. Krachler and W. Shotyk, "Determination of Arsenic in Peat Samples Using HG-AFS and L-Cysteine as Pre-Reductant," Journal of Analytical Atomic Spectrometry, Vol. 21, No. 2, 2006, pp. 204-207. doi: $10.1039 / \mathrm{b} 514268 \mathrm{~g}$

[27] T. Matousek, A. Hernandez-Zavala, M. Svoboda, L. Langrova, B. M. Adair, Z. Drobna, et al., "Oxidation State Specific Generation of Arsines from Methylated Arsenicals Based on L-Cysteine Treatment in Buffered Media for Speciation Analysis by Hydride Generation- 
Automated Cryotrapping-Gas Chromatography-Atomic Absorption Spectrometry with the Multiatomizer," Spectrochimica Acta Part B-Atomic Spectroscopy, Vol. 63, No. 3, 2008, pp. 396-406. doi:10.1016/j.sab.2007.11.037

[28] S. Musil and T. Matousek, "On-Line Pre-Reduction of Pentavalent Arsenicals by Thioglycolic Acid for Speciation Analysis by Selective Hydride Generation-Cryotrapping-Atomic Absorption Spectrometry," Spectrochimica Acta Part B-Atomic Spectroscopy, Vol. 63, No. 6, 2008, pp. 685-691. doi:10.1016/j.sab.2008.04.006

[29] T. Narukawa, T. Kuroiwa, K. Inagaki, A. Takatsu and K. Chiba, "Decomposition of Organoarsenic Compounds for Total Arsenic Determination in Marine Organisms by the Hydride Generation Technique," Applied Organometallic Chemistry, Vol. 19, No. 2, 2005, pp. 239-245. doi:10.1002/aoc.693

[30] C. Lomonte, M. Currell, R. J. S. Morrison, I. D. McKelvie and S. D. Kolev, "Sensitive and Ultra-Fast Determination of Arsenic(III) by Gas-Diffusion Flow Injection Analysis with Chemiluminescence Detection," Analytica Chimica Acta, Vol. 583, No. 1, 2007, pp. 72-77. doi:10.1016/j.aca.2006.09.049

[31] A. Hernandez-Zavala, T. Matousek, Z. Drobna, D. S. Paul, F. Walton, B. M. Adair, et al., "Speciation Analysis of Arsenic in Biological Matrices by Automated Hydride Generation-Cryotrapping-AAS with Multiple Microflame Quartz Tube Atomizer," Journal of Analytical Atomic Spectrometry, Vol. 23, No. 3, 2008, pp. 342-351. doi:10.1039/b706144g

[32] P. Salaun, B. Planer-Friedrich and C. M. G. van den Berg, "Inorganic Arsenic Speciation in Water and Seawater by Anodic Stripping Voltammetry with a Gold Microelectrode," Analytica Chimica Acta, Vol. 585, No. 2, 2007, pp. 312-322. doi:10.1016/j.aca.2006.12.048

[33] B. K. Jena and C. R. Raj, "Gold Nanoelectrode Ensembles for the Simultaneous Electrochemical Detection of Ultratrace Arsenic, Mercury, and Copper," Analytical Chemistry, Vol. 80, No. 13, 2008, pp. 4836-4844. doi:10.1021/ac071064w

[34] M. J. Ruiz-Chancho, J. F. Lopez-Sanchez, E. Schmeisser, W. Goessler, K. A. Francesconi and R. Rubio, "Arsenic Speciation in Plants Growing in Arsenic-Contaminated Sites," Chemosphere, Vol. 71, No. 8, 2008, pp. 15221530. doi:10.1016/j.chemosphere.2007.11.054

[35] J. T. van Elteren, Z. Slejkovec, M. Kahn and W. Goessler, "A Systematic Study on the Extractability of Arsenic Species from Algal Certified Reference Material Iaea-140/Tm (Fucus Sp., Sea Plant Homogenate) Using Methanol/Water Extractant Mixtures," Analytica Chimica Acta, Vol. 585, No. 1, 2007, pp. 24-31. doi:10.1016/j.aca.2006.12.019

[36] P. A. Creed, C. A. Schwegel and J. T. Creed, "Investigation of Arsenic Speciation on Drinking Water Treatment Media Utilizing Automated Sequential Continuous Flow Extraction with IC-ICP-MS Detection," Journal of Environmental Monitoring, Vol. 7, No. 11, 2005, pp. 10791084. doi: $10.1039 / \mathrm{b} 507568 \mathrm{~h}$

[37] K. A. Francesconi, "Complete Extraction of Arsenic Spe- cies: A Worthwhile Goal?" Applied Organometallic Chemistry, Vol. 17, No. 9, 2003, pp. 682-683. doi:10.1002/aoc.499

[38] A. Raab, D. R. Genney, A. A. Meharg and J. Feldmann, "Identification of Arsenic Species in Sheep-Wool Extracts by Different Chromatographic Methods," Applied Organometallic Chemistry, Vol. 17, No. 9, 2003, pp. 684-692. doi:10.1002/aoc.488

[39] H. Hasegawa, M. Matsui, S. Okamura, M. Hojo, N. Iwasaki and Y. Sohrin, "Arsenic Speciation Including 'Hidden' Arsenic in Natural Waters," Applied Organometallic Chemistry, Vol. 13, No. 2, 1999, pp. 113-119. doi:10.1002/(SICI)1099-0739(199902)13:2<113::AID-A OC837>3.0.CO;2-A

[40] G. E. M. Hall, J. C. Pelchat and G. Gauthier, "Stability of Inorganic Arsenic (III) and Arsenic (V) in Water Samples," Journal of Analytical Atomic Spectrometry, Vol. 14, No. 2, 1999, pp. 205-213. doi:10.1039/a807498d

[41] B. Planer-Friedrich, J. C. Fisher, J. T. Hollibaugh, E. Suss and D. Wallschlager, "Oxidative Transformation of Trithioarsenate Along Alkaline Geothermal Drainages Abiotic Versus Microbially Mediated Processes," Geomicrobiology Journal, Vol. 26, No. 5, 2009, pp. 339-350. doi:10.1080/01490450902755364

[42] D. Wallschlager and J. London, "Determination of Methylated Arsenic-Sulfur Compounds in Groundwater," Environmental Science \& Technology, Vol. 42, No. 1, 2008, pp. 228-234. doi:10.1021/es0707815

[43] S. D. Conklin, M. W. Fricke, P. A. Creed and J. T. Creed, "Investigation of the Ph Effects on the Formation of Methylated Thio-Arsenicals, and the Effects of $\mathrm{pH}$ and Temperature on Their Stability," Journal of Analytical Atomic Spectrometry, Vol. 23, No. 5, 2008, pp. 711-716. doi: $10.1039 / \mathrm{b} 713145 \mathrm{c}$

[44] R. Wahlen, S. McSheehy, C. Scriver and Z. Mester, “Arsenic Speciation in Marine Certified Reference Materials - Part 2. The Quantification of Water-Soluble Arsenic Species by HPLC-ICP MS," Journal of Analytical Atomic Spectrometry, Vol. 19, No. 7, 2004, pp. 876-882. doi: $10.1039 / \mathrm{b} 402482 \mathrm{f}$

[45] S. McSheehy and Z. Mester, "Arsenic Speciation in Marine Certified Reference Materials - Part 1. Identification of Water-Soluble Arsenic Species Using Multidimensional Liquid Chromatography Combined with Inductively Coupled Plasma, Electrospray and Electrospray High-Field Asymmetric Waveform Ion Mobility Spectrometry with Mass Spectrometric Detection," Journal of Analytical Atomic Spectrometry, Vol. 19, No. 3, 2004, pp. 373-380. doi:10.1039/b314101b

[46] J. Zheng and H. Hintelmann, "HPLC-ICP-MS for a Comparative Study on the Extraction Approaches for Arsenic Speciation in Terrestrial Plant, Ceratophyllum Demersum," Journal of Radioanalytical and Nuclear Chemistry, Vol. 280, No. 1, 2009, pp. 171-179. doi:10.1007/s10967-008-7440-Z

[47] A. C. Schmidt, N. Haufe and M. Otto, "A Systematic Study on Extraction of Total Arsenic from Down-Scaled Sample Sizes of Plant Tissues and Implications for Arse- 
nic Species Analysis," Talanta, Vol. 76, No. 5, 2008, pp. 1233-1240. doi:10.1016/j.talanta.2008.05.032

[48] K. A. Mir, A. Rutter, I. Koch, P. Smith, K. J. Reimer and J. S. Poland, "Extraction and Speciation of Arsenic in Plants Grown on Arsenic Contaminated Soils," Talanta, Vol. 72, No. 4, 2007, pp. 1507-1518. doi:10.1016/j.talanta.2007.01.068

[49] R. Rubio, M. J. Ruiz-Chancho, J. F. López-Sánchez, R. Rubio and J. F. López-Sánchez, "Sample Pre-Treatment and Extraction Methods That Are Crucial to Arsenic Speciation in Algae and Aquatic Plants," TrAC- Trends in Analytical Chemistry, Vol. 29, No. 1, 2010, pp. 53-69. doi:10.1016/j.trac.2009.10.002

[50] C. G. Yuan, B. He, E. L. Gao, J. X. Lu and G. B. Jiang, "Evaluation of Extraction Methods for Arsenic Speciation in Polluted Soil and Rotten Ore by HPLC-HG-AFS Analysis," Microchimica Acta, Vol. 159, No. 1-2, 2007, pp. 175-182. doi:10.1007/s00604-006-0709-4

[51] K. H. Goh and T. T. Lim, "Arsenic Fractionation in a Fine Soil Fraction and Influence of Various Anions on Its Mobility in the Subsurface Environment," Applied Geochemistry, Vol. 20, No. 2, 2005, pp. 229-239. doi:10.1016/j.apgeochem.2004.08.004

[52] I. Pizarro, M. Gomez, C. Camara and M. A. Palacios, "Arsenic Speciation in Environmental and Biological Samples - Extraction and Stability Studies," Analytica Chimica Acta, Vol. 495, No. 1-2, 2003, pp. 85-98. doi:10.1016/j.aca.2003.08.009

[53] M. M. Rahman, Z. L. Chen and R. Naidu, "Extraction of Arsenic Species in Soils Using Microwave-Assisted Extraction Detected by Ion Chromatography Coupled to ICP MS," Environmental Geochemistry and Health, Vol. 31, 2009, pp. 93-102. doi:10.1007/s10653-008-9227-0

[54] F. Rahman, Z. L. Chen and R. Naidu, "A Comparative Study of the Extractability of Arsenic Species from Silverbeet and Amaranth Vegetables," Environmental Geochemistry and Health, Vol. 31, 2009, pp. 103-113. doi:10.1007/s10653-008-9225-2

[55] E. Smith, A. L. Juhasz and J. Weber, "Arsenic Uptake and Speciation in Vegetables Grown under Greenhouse Conditions," Environmental Geochemistry and Health, Vol. 31, 2009, pp. 125-132. doi:10.1007/s10653-008-9242-1

[56] R. E. Rivas, I. Lopez-Garcia and M. Hernandez-Cordoba, "Speciation of Very Low Amounts of Arsenic and Antimony in Waters Using Dispersive Liquid-Liquid Microextraction and Electrothermal Atomic Absorption Spectrometry," Spectrochimica Acta Part B-Atomic Spectroscopy, Vol. 64, No. 4, 2009, pp. 329-333. doi:10.1016/j.sab.2009.03.007

[57] K. H. Al-Assaf, J. F. Tyson and P. C. Uden, "Determination of Four Arsenic Species in Soil by Sequential Extraction and High Performance Liquid Chromatography with Post-Column Hydride Generation and ICP Optical Emission Spectrometry Detection," Journal of Analytical Atomic Spectrometry, Vol. 24, No. 4, 2009, pp. 376-384. doi:10.1039/b820300h

[58] M. N. M. Reyes, M. L. Cervera, R. C. Campos and M. de la Guardia, "Non-Chromatographic Speciation of Toxic Arsenic in Vegetables by HG AFS after Ultrasound-Assisted Extraction," Talanta, Vol. 75, No. 3, 2008, pp. 811-816. doi:10.1016/j.talanta.2007.12.018

[59] V. Dufailly, L. Noel, J. M. Fremy, D. Beauchemin and T. Guerin, "Optimisation by Experimental Design of an IEC/ICP-MS Speciation Method for Arsenic in Seafood Following Microwave Assisted Extraction," Journal of Analytical Atomic Spectrometry, Vol. 22, No. 9, 2007, pp. 1168-1173. doi:10.1039/b705798a

[60] V. Kaur, A. K. Malik and N. Verma, "Applications of Solid Phase Microextraction for the Determination of Metallic and Organometallic Species," Journal of Separation Science, Vol. 29, No. 3, 2006, pp. 333-345. doi:10.1002/jssc. 200500319

[61] Z. Mester and R. Sturgeon, "Trace Element Speciation Using Solid Phase Microextraction," Spectrochimica Acta Part B-Atomic Spectroscopy, Vol. 60, No. 9-10, 2005, pp. 1243-1269. doi:10.1016/j.sab.2005.06.013

[62] Z. Mester, R. Sturgeon and J. Pawliszyn, "Solid Phase Microextraction as a Tool for Trace Element Speciation," Spectrochimica Acta Part B-Atomic Spectroscopy, Vol. 56, No. 3, 2001, pp. 233-260. doi:10.1016/S0584-8547(00)00304-9

[63] M. Mulugeta, G. Wibetoe, C. J. Engelsen and W. Lund, "Speciation Analysis of as, $\mathrm{Sb}$ and Se in Leachates of Cementitious Construction Materials Using Selective Solid Phase Extraction and ICP-MS," Journal of Analytical Atomic Spectrometry, Vol. 25, No. 2, 2010, pp. 169-177. doi:10.1039/b919994m

[64] X. L. Liu, T. C. Duan, Y. Han, X. Y. Jia and H. T. Chen, "On-Line Solid Phase Extraction-Hydride Generation Atomic Fluorescence Spectrometric Determination of Trace Arsenic in High Purity Antimony(III) Oxide," Journal of Analytical Atomic Spectrometry, Vol. 25, No. 2, 2010, pp. 206-209. doi:10.1039/b915054d

[65] Z. Slejkovec, I. Falnoga, W. Goessler, J. T. van Elteren, R. Raml, H. Podgornik, et al., "Analytical Artefacts in the Speciation of Arsenic in Clinical Samples," Analytica Chimica Acta, Vol. 607, No. 1, 2008, pp. 83-91. doi:10.1016/j.aca.2007.11.031

[66] S. G. Salgado, M. A. Q. Nieto and M. M. B. Simón, “Assessment of Total Arsenic and Arsenic Species Stability in Alga Samples and Their Aqueous Extracts," Talanta, Vol. 75, No. 4, 2008, pp. 897-903. doi:10.1016/j.talanta.2007.12.031

[67] V. Oliveira, A. M. Sarmiento, J. L. Gomez-Ariza, J. M. Nieto and D. Sanchez-Rodas, "New Preservation Method for Inorganic Arsenic Speciation in Acid Mine Drainage Samples," Talanta, Vol. 69, No. 5, 2006, pp. 1182-1189. doi:10.1016/j.talanta.2005.12.034

[68] A. G. Gault, J. Jana, S. Chakraborty, P. Mukherjee, M. Sarkar, B. Nath, et al., "Preservation Strategies for Inorganic Arsenic Species in High Iron, Low-Eh Groundwater from West Bengal, India," Analytical and Bioanalytical Chemistry, Vol. 381, No. 2, 2005, pp. 347-353. doi:10.1007/s00216-004-2861-1

[69] R. B. McCleskey, D. K. Nordstrom and A. S. Maest, 
"Preservation of Water Samples for Arsenic(III/V) Determinations: An Evaluation of the Literature and New Analytical Results," Applied Geochemistry, Vol. 19, No. 7, 2004, pp. 995-1009.

doi:10.1016/j.apgeochem.2004.01.003

[70] B. Daus, H. Weiss, J. Mattusch and R. Wennrich, "Preservation of Arsenic Species in Water Samples Using Phosphoric Acid - Limitations and Long-Term Stability," Talanta, Vol. 69, 2006, pp. 430-434. doi:10.1016/j.talanta.2005.10.012

[71] B. Daus, J. Mattusch, R. Wennrich and H. Weiss, "Investigation on Stability and Preservation of Arsenic Species in Iron Rich Water Samples," Talanta, Vol. 58, No. 1, 2002, pp. 57-65. doi:10.1016/S0039-9140(02)00256-4

[72] A. J. Bednar, J. R. Garbarino, M. R. Burkhardt, J. F. Ranville and T. R. Wildeman, "Field and Laboratory Arsenic Speciation Methods and Their Application to Natural-Water Analysis," Water Research, Vol. 38, No. 2, 2004, pp. 355-364. doi:10.1016/j.watres.2003.09.034

[73] A. J. Bednar, J. R. Garbarino, J. F. Ranville and T. R. Wildeman, "Preserving the Distribution of Inorganic Arsenic Species in Groundwater and in Acid Mine Drainage Samples," Environmental Science \& Technology, Vol. 36, 2002, pp. 2213-2218. doi:10.1021/es0157651

[74] P. A. Gallagher, C. A. Schwegel, X. Y. Wei and J. T. Creed, "Speciation and Preservation of Inorganic Arsenic in Drinking Water Sources Using EDTA with IC Separation and ICP-MS Detection," Journal of Environmental Monitoring, Vol. 3, No. 4, 2001, pp. 371-376. doi:10.1039/b101658j

[75] V. Dufailly, T. Guerin, L. Noel, J. M. Fremy and D. Beauchemin, "A Simple Method for the Speciation Analysis of Bio-Accessible Arsenic in Seafood Using On-Line Continuous Leaching and Ion Exchange Chromatography Coupled to ICP MS," Journal of Analytical Atomic Spectrometry, Vol. 23, No. 9, 2008, pp. 12631268. doi:10.1039/b803516d

[76] M. Rees, L. Sansom, A. Rofe, A. L. Juhasz, E. Smith, J. Weber, et al., "Principles and Application of an in Vivo Swine Assay for the Determination of Arsenic Bioavailability in Contaminated Matrices," Environmental Geochemistry and Health, Vol. 31, 2009, pp. 167-177. doi:10.1007/s10653-008-9237-y

[77] M. Ponthieu, P. Pinel-Raffaitin, I. Le Hecho, L. Mazeas, D. Amouroux, O. F. X. Donard, et al., "Speciation Analysis of Arsenic in Landfill Leachate," Water Research, Vol. 41, No. 14, 2007, pp. 3177-3185. doi:10.1016/j.watres.2007.04.026

[78] P. Pinel-Raffaitin, I. Le Hecho, D. Amouroux and M. Potin-Gautter, "Distribution and Fate of Inorganic and Organic Arsenic Species in Landfill Leachates and Biogases," Environmental Science \& Technology, Vol. 41, No. 13, 2007, pp. 4536-4541. doi:10.1021/es0628506

[79] J. Szpunar, S. McSheehy, K. Polec, V. Vacchina, S. Mounicou, I. Rodriguez, et al., "Gas and Liquid Chromatography with Inductively Coupled Plasma Mass Spectrometry Detection for Environmental Speciation Analysis - Advances and Limitations," Spectrochimica
Acta Part B-Atomic Spectroscopy, Vol. 55, No. 7, 2000, pp. 779-793. doi:10.1016/S0584-8547(00)00210-X

[80] A. Raab, J. Feldmann and A. A. Meharg, "The Nature of Arsenic-Phytochelatin Complexes in Holcus Lanatus and Pteris Cretica," Plant Physiology, Vol. 134, No. 3, 2004, pp. 1113-1122. doi:10.1104/pp.103.033506

[81] B. K. Mandal, K. T. Suzuki, K. Anzai, K. Yamaguchi and Y. Sei, "A SEC-HPLC-ICP MS Hyphenated Technique for Identification of Sulfur-Containing Arsenic Metabolites in Biological Samples," Journal of Chromatography B-Analytical Technologies in the Biomedical and Life Sciences, Vol. 874, No. 1-2, 2008, pp. 64-76. doi:10.1016/j.jchromb.2008.09.004

[82] D. Melamed, "Monitoring Arsenic in the Environment: A Review of Science and Technologies with the Potential for Field Measurements," Analytica Chimica Acta, Vol. 532, No. 1, 2005, pp. 1-13. doi:10.1016/j.aca.2004.10.047

[83] C. M. Steinmaus, C. M. George, D. A. Kalman and A. H. Smith, "Evaluation of Two New Arsenic Field Test Kits Capable of Detecting Arsenic Water Concentrations Close to $10 \mu \mathrm{g} / \mathrm{L}$," Environmental Science \& Technology, Vol. 40, No. 10, 2006, pp. 3362-3366. doi:10.1021/es060015i

[84] N. A. Yusof and K. Rashid, "Development of Optical Test Strip for Rapid Determination of Trace Arsenic Using Immobilized Gallocyanine," Asian Journal of Chemistry, Vol. 21, No. 3, 2009, pp. 1747-1753.

[85] M. Arora, M. Megharaj and R. Naidu, "Arsenic Testing Field Kits: Some Considerations and Recommendations," Environmental Geochemistry and Health, Vol. 31, 2009, pp. 45-48. doi:10.1007/s10653-008-9231-4

[86] D. G. Kinniburgh and W. Kosmus, "Arsenic Contamination in Groundwater: Some Analytical Considerations," Talanta, Vol. 58, No. 1, 2002, pp. 165-180. doi:10.1016/S0039-9140(02)00265-5

[87] E. Diesel, M. Schreiber and J. R. van der Meer, "Development of Bacteria-Based Bioassays for Arsenic Detection in Natural Waters," Analytical and Bioanalytical Chemistry, Vol. 394, No. 3, 2009, pp. 687-693. doi:10.1007/s00216-009-2785-x

[88] J. Z. Xing, L. J. Zhu, J. A. Jackson, S. Gabos, X. J. Sun, X. B. Wang, et al., "Dynamic Monitoring of Cytotoxicity on Microelectronic Sensors," Chemical Research in Toxicology, Vol. 18, No. 2, 2005, pp. 154-161. doi:10.1021/tx049721s

[89] R. Liu, P. Wu, M. Y. Xi, K. L. Xu and Y. Lv, "Inorganic Arsenic Speciation Analysis of Water Samples by Trapping Arsine on Tungsten Coil for Atomic Fluorescence Spectrometric Determination," Talanta, Vol. 78, No. 3, 2009, pp. 885-890. doi:10.1016/j.talanta.2008.12.067

[90] J. Gomez-Ariza, F. Lorenzo and T. Garcia-Barrera, "Comparative Study of Atomic Fluorescence Spectroscopy and ICP MS for Mercury and Arsenic Multispeciation," Analytical and Bioanalytical Chemistry, Vol. 382, No. 2, 2005, pp. 485-492. doi:10.1007/s00216-005-3094-7

[91] B. Chen, M. Krachler, Z. I. Gonzalez and W. Shotyk, 
"Improved Determination of Arsenic in Environmental and Geological Specimens Using HG-AFS," Journal of Analytical Atomic Spectrometry, Vol. 20, No. 2, 2005, pp. 95-102. doi:10.1039/b416142d

[92] E. Munoz and S. Palmero, "Analysis and Speciation of Arsenic by Stripping Potentiometry: A Review," Talanta, Vol. 65, No. 3, 2005, pp. 613-620. doi:10.1016/j.talanta.2004.07.034

[93] A. Cavicchioli, M. A. La-Scalea and I. G. R. Gutz, "Analysis and Speciation of Traces of Arsenic in Environmental Food and Industrial Samples by Voltammetry: A Review," Electroanalysis, Vol. 16, No. 9, 2004, pp. 697-711. doi:10.1002/elan.200302936

[94] D. Beauchemin, "Inductively Coupled Plasma Mass Spectrometry," Analytical Chemistry, Vol. 80, No. 12, 2008, pp. 4455-4486. doi:10.1021/ac8006945

[95] K. L. Ackley, K. L. Sutton and J. A. Caruso, "The Use of ICP-MS as a Detector for Elemental Speciation Studies Comprehensive Analytical Chemistry," In: J. A. Caruso, K. L. Sutton and K. L. Ackley, Eds., Elemental Speciation New Approach for Trace Element Analysis, Elsevier, Vol. 33, 2000, pp. 249-276.

[96] R. Cornelis, J. Caruso, H. Crews and K. Heumann, Handbook of Elemental Speciation: Techniques and Methodology, John Wiley \& Sons, Chichester, 2003. doi: $10.1002 / 0470868384$

[97] R. Lobinski, D. Schaumlöffel and J. Szpunar, "Mass Spectrometry in Bioinorganic Analytical Chemistry," Mass Spectrometry Reviews, Vol. 25, No. 2, 2006, pp. 255-289. doi:10.1002/mas.20069

[98] M. Moldovan, E. M. Krupp, A. E. Holliday and O. F. X. Donard, "High Resolution Sector Field ICP-MS and Multicollector ICP-MS as Tools for Trace Metal Speciation in Environmental Studies: A Review," Journal of Analytical Atomic Spectrometry, Vol. 19, No. 7, 2004, pp. 815-822. doi:10.1039/b403128h

[99] A. A. Ammann, "Inductively Coupled Plasma Mass Spectrometry (ICP-MS): A Versatile Tool," Journal of Mass Spectrometry, Vol. 42, No. 4, 2007, pp. 419-427. doi:10.1002/jms.1206

[100] J. Feldmann, "What Can the Different Current-Detection Methods Offer for Element Speciation?" TrAC-Trends in Analytical Chemistry, Vol. 24, No. 3, 2005, pp. 228-242. doi:10.1016/j.trac.2004.11.011

[101]Z. L. Zhu and Q. Qin, "Recent Development of Speciation Analysis for Trace Arsenic," Spectroscopy and Spectral Analysis, Vol. 28, No. 5, 2008, pp. 1176-1180.

[102] A. V. Hirner, "Speciation of Alkylated Metals and Metalloids in the Environment," Analytical and Bioanalytical Chemistry, Vol. 385, No. 3, 2006, pp. 555-567. doi:10.1007/s00216-006-0368-7

[103] F. C. Adams, "Elemental Speciation: Where Do We Come From? Where Do We Go?" Journal of Analytical Atomic Spectrometry, Vol. 19, No. 9, 2004, pp. 1090-1097. doi:10.1039/b400559g

[104] S. Choi, P. A. O'Day and J. G. Hering, "Natural Attenuation of Arsenic by Sediment Sorption and Oxidation,"
Environmental Science \& Technology, Vol. 43, No. 12, 2009, pp. 4253-4259. doi:10.1021/es802841x

[105] M. Grafe, R. V. Tappero, M. A. Marcus and D. L. Sparks, "Arsenic Speciation in Multiple Metal Environments: I. Bulk-XAFS Spectroscopy of Model and Mixed Compounds," Journal of Colloid and Interface Science, Vol. 320, No. 2, 2008, pp. 383-399. doi:10.1016/j.jcis.2008.01.028

[106] R. T. Wilkin and R. G. Ford, "Arsenic Solid-Phase Partitioning in Reducing Sediments of a Contaminated Wetland," Chemical Geology, Vol. 228, No. 1-3, 2006, pp. 156-174. doi:10.1016/j.chemgeo.2005.11.022

[107] A. G. Gault, D. A. Polya, P. R. Lythgoe, M. L. Farquhar, J. M. Charnock and R. A. Wogelius, "Arsenic Speciation in Surface Waters and Sediments in a Contaminated Waterway: An IC ICP-MS and XAS Based Study," Applied Geochemistry, Vol. 18, No. 9, 2003, pp. 1387-1397. doi:10.1016/S0883-2927(03)00058-1

[108] C. G. Yuan and X. C. Le, "Arsenic Speciation Analysis," Progress in Chemistry, Vol. 21, No. 2-3, 2009, pp. 467-473.

[109] M. Leermakers, W. Baeyens, M. De Gieter, B. Smedts, C. Meert, H. C. De Bisschop, et al., "Toxic Arsenic Compounds in Environmental Samples: Speciation and Validation," TrAC-Trends in Analytical Chemistry, Vol. 25, No. 1, 2006, pp. 1-10. doi:10.1016/j.trac.2005.06.004

[110] E. Terlecka, "Arsenic Speciation Analysis in Water Samples: A Review of the Hyphenated Techniques," Environmental Monitoring and Assessment, Vol. 107, No. 1-3, 2005, pp. 259-284. doi:10.1007/s10661-005-3109-Z

[111] G. Raber, S. Khoomrung, M. S. Taleshi, J. S. Edmonds and K. A. Francesconi, "Identification of Arsenolipids with GC/MS," Talanta, Vol. 78, No. 3, 2009, pp. 1215-1218. doi:10.1016/j.talanta.2009.01.013

[112]R. A. Diaz-Bone, M. Hollmann, O. Wuerfel and D. Pieper, "Analysis of Volatile Arsenic Compounds Formed by Intestinal Microorganisms: Rapid Identification of New Metabolic Products by Use of Simultaneous EI-MS and ICP-MS Detection after Gas Chromatographic Separation," Journal of Analytical Atomic Spectrometry, Vol. 24, No. 6, 2009, pp. 808-814. doi: $10.1039 / \mathrm{b} 822968 \mathrm{f}$

[113] E. Schmeisser, W. Goessler, N. Kienzl and K. A. Francesconi, "Volatile Analytes Formed from Arsenosugars: Determination by HPLC-HG-ICP-MS and Implications for Arsenic Speciation Analyses," Analytical Chemistry, Vol. 76, No. 2, 2004, pp. 418-423. doi: $10.1021 / \mathrm{ac} 034878 \mathrm{v}$

[114] R. Regmi, B. F. Milne and J. Feldmann, "Hydride Generation Activity of Arsenosugars and Thioarsenicals," Analytical and Bioanalytical Chemistry, Vol. 388, No. 4, 2007, pp. 775-782. doi:10.1007/s00216-006-1076-z

[115] N. Campillo, R. Penalver, P. Vinas, I. Lopez-Garcia and M. Hernandez-Cordoba, "Speciation of Arsenic Using Capillary Gas Chromatography with Atomic Emission Detection," Talanta, Vol. 77, No. 2, 2008, pp. 793-799. doi:10.1016/j.talanta.2008.07.028

[116] J. C. A. Wuilloud, R. G. Wuilloud, A. P. Vonderheide 
and J. A. Caruso, "Gas Chromatography/Plasma Spectrometry - An Important Analytical Tool for Elemental Speciation Studies," Spectrochimica Acta Part B-Atomic Spectroscopy, Vol. 59, No. 6, 2004, pp. 755-792. doi:10.1016/j.sab.2004.03.009

[117] B. G. Sun, M. Macka and P. R. Haddad, "Trace Determination of Arsenic Species by Capillary Electrophoresis with Direct UV Detection Using Sensitivity Enhancement by Counter- or Co-Electroosmotic Flow Stacking and a High-Sensitivity Cell," Electrophoresis, Vol. 24, No. 12-13, 2003, pp. 2045-2053. doi:10.1002/elps.200305447

[118] O. S. Koshcheeva, O. V. Shuvaeva and L. I. Kuznetzova, "Arsenic Speciation in Natural and Contaminated Waters Using CZE with in Situ Derivatization by Molybdate and Direct UV-Detection," Electrophoresis, Vol. 30, No. 6, 2009, pp. 1088-1093. doi:10.1002/elps.200800384

[119] K. Kutschera, A. C. Schmidt, S. Kohler and M. Otto, "CZE for the Speciation of Arsenic in Aqueous Soil Extracts," Electrophoresis, Vol. 28, No. 19, 2007, pp. 3466-3476. doi:10.1002/elps.200700107

[120] F. Kitagawa, K. Shiomi and K. Otsuka, "Analysis of Arsenic Compounds by Capillary Electrophoresis Using Indirect UV and Mass Spectrometric Detections," Electrophoresis, Vol. 27, No. 11, 2006, pp. 2233-2239. doi:10.1002/elps.200500614

[121] K. F. Akter, Z. Chen, L. Smith, D. Davey and R. Naidu, "Speciation of Arsenic in Ground Water Samples: A Comparative Study of CE-UV, HG-AAS and LC ICP MS," Talanta, Vol. 68, No. 2, 2005, pp. 406-415. doi:10.1016/j.talanta.2005.09.011

[122] B. Michalke, "Capillary Electrophoresis ICP MS: A Report on Technical Principles and Problem Solutions, Potential, and Limitations of This Technology as Well as on Examples of Application," Electrophoresis, Vol. 26, No. 7-8, 2005, pp. 1584-1597. doi:10.1002/elps.200410314

[123] C. Casiot, O. F. X. Donard and M. Potin-Gautier, "Optimization of the Hyphenation between Capillary Zone Electrophoresis and ICP MS for the Measurement of As-, Sb-, Se- and Te-Species, Applicable to Soil Extracts," Spectrochimica Acta Part B-Atomic Spectroscopy, Vol. 57, No. 1, 2002, pp. 173-187. doi:10.1016/S0584-8547(01)00365-2

[124] G. D. Yang, J. H. Xu, J. P. Zheng, X. Q. Xu, W. Wang, L. J. Xu, et al., "Speciation Analysis of Arsenic in Mya Arenaria Linnaeus and Shrimp with Capillary Electrophoresis-Inductively Coupled Plasma Mass Spectrometry," Talanta, Vol. 78, No. 2, 2009, pp. 471-476. doi:10.1016/j.talanta.2008.11.040

[125] G. Koellensperger, J. Nurmi, S. Hann, G. Stingeder, W. J. Fitz and W. W. Wenzel, "CE-ICP-SFMS and HPLC ICP SFMS for Arsenic Speciation in Soil Solution and Soil Water Extracts," Journal of Analytical Atomic Spectrometry, Vol. 17, No. 9, 2002, pp. 1042-1047. doi: $10.1039 / \mathrm{b} 202875 \mathrm{c}$

[126] E. H. Larsen, "Method Optimization and Quality Assurance in Speciation Analysis Using HPLC with Detection by ICP MS," Spectrochimica Acta Part B-Atomic Spec- troscopy, Vol. 53, No. 2, 1998, pp. 253-265. doi:10.1016/S0584-8547(97)00137-7

[127] J. Weiss and T. Weiss, Handbook of Ion Chromatography, 3rd Edition, Weinheim: Wiley-VCH, 2004. doi:10.1002/9783527619610

[128] J. S. Fritz and D. T. Gjerde, Ion Chromatography, 4th Edition, Weinheim: Wiley, 2009. doi:10.1002/9783527626878

[129] M. D. Zimmermann and J. A. Tossell, "Acidities of Arsenic (III) and Arsenic (V) Thio- and Oxyacids in Aqueous Solution Using the CBS-QB3/CPCM Method," Journal of Physical Chemistry A, Vol. 113, No. 17, 2009, pp. 5105-5111. doi:10.1021/jp809123q

[130] A. A. Ammann, “Arsenic Speciation by Gradient Anion Exchange Narrow Bore Ion Chromatography and High Resolution ICP MS Detection," Journal of Chromatography A, Vol. 1217, 2010, pp. 2111-2116. doi:10.1016/j.chroma.2010.01.086

[131] M. Morita, T. Uehiro and K. Fuwa, "Detremination of Arsenic Compounds in Biological Samples by Liquid Chromatography with Inductively Coupled Argon PlasmaEmission Spectrometric Detection," Analytical Chemistry, Vol. 53, No. 12, 1981, pp. 1806-1808. doi:10.1021/ac00235a021

[132] A. K. Malik, M. Gomez, C. Camara, H. G. Riepe and J. Bettmer, "On-Line Chloride Interference Removal for Arsenic Determination in Waste Water and Urine by ICP MS Using a Modified Capillary," International Journal of Environmental Analytical Chemistry, Vol. 82, No. 11, 2002, pp. 795-804. doi:10.1080/0306731021000102266

[133] L. Yehiayan, M. Pattabiraman, K. Kavallieratos, X. T. Wang, L. H. Boise and Y. Cai, "Speciation, Formation, Stability and Analytical Challenges of Human Arsenic Metabolites," Journal of Analytical Atomic Spectrometry, Vol. 24, No. 10, 2009, pp. 1397-1405. doi:10.1039/b910943a

[134]Z. Gong, X. Lu, M. Ma, C. Watt and X. C. Le, “Arsenic Speciation Analysis," Talanta, Vol. 58, No. 1, 2002, pp. 77-96. doi:10.1016/S0039-9140(02)00258-8

[135] P. Teräsahde, M. Pantsar-Kallio and P. K. G. Manninen, "Simultaneous Determination of Arsenic Species by Ion Chromatography ICP MS," Journal of Chromatography A, Vol. 750, No. 1-2, 1996, pp. 83-88. doi:10.1016/0021-9673(96)00469-4

[136] T. Sakai, Y. Inoue, Y. Date, T. Aoyama, K. Yoshida and G. Endo, "Simultaneous Determination of Neutral, Anionic and Cationic Compounds within One Chromatographic Run Using an ICP MS as Element-Specific Detector," Applied Organometallic Chemistry, Vol. 15, No. 4, 2001, pp. 285-290. doi:10.1002/aoc.141

[137] T. Cecchi, "Ion Pairing Chromatography," Critical Reviews in Analytical Chemistry, Vol. 38, No. 3, 2008, pp. 161-213. doi:10.1080/10408340802038882

[138] S. H. Hansen, E. H. Larsen, G. Pritzl and C. Cornett, "Separation of Seven Arsenic Compounds by High-Performance Liquid-Chromatography with Online Detection by Hydrogen-Argon Flame Atomic Absorption Spectrometry and ICP MS," Journal of Analytical Atomic 
Spectrometry, Vol. 7, No. 4, 1992, pp. 629-634. doi:10.1039/ja9920700629

[139] D. Beauchemin, K. W. M. Siu, J. W. McLaren and S. S. Berman, "Determination of Arsenic Species by High-Performance Liquid-Chromatography - ICP MS," Journal of Analytical Atomic Spectrometry, Vol. 4, No. 3, 1989, pp. 285-289. doi:10.1039/ja9890400285

[140] S. Wangkarn and S. A. Pergantis, "High-Speed Separation of Arsenic Compounds Using Narrow-Bore HighPerformance Liquid Chromatography On-Line with ICP MS," Journal of Analytical Atomic Spectrometry, Vol. 15, No. 6, 2000, pp. 627-633. doi:10.1039/b001810o

[141] S. Afton, K. Kubachka, B. Catron and J. A. Caruso, "Simultaneous Characterization of Selenium and Arsenic Analytes Via Ion-Pairing Reversed Phase Chromatography with Inductively Coupled Plasma and Electrospray Ionization Ion Trap Mass Spectrometry for Detection Applications to River Water, Plant Extract and Urine Matrices," Journal of Chromatography A, Vol. 1208, No. 1-2, 2008, pp. 156-163.

doi:10.1016/j.chroma.2008.08.077

[142] J. Mattusch and R. Wennrich, "Determination of Anionic, Neutral, and Cationic Species of Arsenic by Ion Chromatography with ICP MS Detection in Environmental Samples," Analytical Chemistry, Vol. 70, No. 17, 1998, pp. 3649-3655. doi:10.1021/ac9802574

[143] S. Londesborough, J. Mattusch and R. Wennrich, "Separation of Organic and Inorganic Arsenic Species by HPLC ICP MS," Fresenius Journal of Analytical Chemistry, Vol. 363, No. 5-6, 1999, pp. 577-581. doi:10.1007/s002160051251

[144] B. P. Jackson and P. M. Bertsch, "Determination of Arsenic Speciation in Poultry Wastes by IC ICP MS," Environmental Science \& Technology, Vol. 35, No. 24, 2001, pp. 4868-4873. doi:10.1021/es0107172

[145] U. Kohlmeyer, E. Jantzen, J. Kuballa and S. Jakubik, "Benefits of High Resolution IC ICP MS for the Routine Analysis of Inorganic and Organic Arsenic Species in Food Products of Marine and Terrestrial Origin," Analytical and Bioanalytical Chemistry, Vol. 377, No. 1, 2003, pp. 6-13. doi:10.1007/s00216-003-2064-1

[146] U. Kohlmeyer, J. Kuballa and E. Jantzen, "Simultaneous Separation of 17 Inorganic and Organic Arsenic Compounds in Marine Biota by Means of HPLC ICP MS," Rapid Communications in Mass Spectrometry, Vol. 16, No. 10, 2002, pp. 965-974. doi:10.1002/rcm.671

[147] S. Simon, H. Tran, F. Pannier and M. Potin-Gautier, "Simultaneous Determination of Twelve Inorganic and Organic Arsenic Compounds by Liquid Chromatography-Ultraviolet Irradiation-Hydride Generation Atomic Fluorescence Spectrometry," Journal of Chromatography A, Vol. 1024, No. 1-2, 2004, pp. 105-113. doi:10.1016/j.chroma.2003.09.068

[148] B. Daus, J. Mattusch, A. Paschke, R. Wennrich and H. Weiss, "Kinetics of the Arsenite Oxidation in Seepage Water from a Tin Mill Tailings Pond," Talanta, Vol. 51, No. 6, 2000, pp. 1087-1095. doi:10.1016/S0039-9140(00)00302-7
[149] P. Zbinden, D. Andrey and C. Blake, "A Routine Ion Chromatography ICP MS Method for the Analysis of Arsenic Species Applicable in the Food Industry," Atomic Spectroscopy, Vol. 21, No. 6, 2000, pp. 205-215.

[150] A. Raab, A. A. Meharg, M. Jaspars, D. R. Genney and J. Feldmann, "Arsenic-Glutathione Complexes - Their Stability in Solution and During Separation by Different HPLC Modes," Journal of Analytical Atomic Spectrometry, Vol. 19, No. 1, 2004, pp. 183-190. doi: $10.1039 / \mathrm{b} 307945 \mathrm{~g}$

[151] S. Fitzpatrick, L. Ebdon and M. E. Foulkes, "Separation and Detection of Arsenic and Selenium Species in Environmental Samples by HPLC ICP MS," International Journal of Environmental Analytical Chemistry, Vol. 82, No. 11-12, 2002, pp. 835-841. doi:10.1080/03067310290013267

[152] B. A. Manning and D. A. Martens, "Speciation of Arsenic (III) and Arsenic (V) in Sediment Extracts by HPLC HG AAS," Environmental Science \& Technology, Vol. 31, No. 1, 1997, pp. 171-177. doi:10.1021/es9602556

[153] S. Polesello, S. Valsecchi, S. Cavalli and C. Reschiotto, "Ion-Chromatographic Screening Method for Monitoring Arsenate and Other Anionic Pollutants in Ground Waters of Northern Italy," Journal of Chromatography A, Vol. 920, No. 1-2, 2001, pp. 231-238. doi:10.1016/S0021-9673(01)00745-2

[154] J. A. Brisbin, C. B'Hymer and J. A. Caruso, “A Gradient Anion Exchange Chromatographic Method for the Speciation of Arsenic in Lobster Tissue Extracts," Talanta, Vol. 58, No. 1, 2002, pp. 133-145. doi:10.1016/S0039-9140(02)00262-X

[155] R. Xie, W. Johnson, S. Spayd, G. S. Hall and B. Buckley, "Arsenic Speciation Analysis of Human Urine Using Ion Exchange Chromatography Coupled to ICP MS," Analytica Chimica Acta, Vol. 578, No. 2, 2006, pp. 186-194. doi:10.1016/j.aca.2006.06.076

[156] T. Lindemann, A. Prange, W. Dannecker and B. Neidhart, "Simultaneous Determination of Arsenic, Selenium and Antimony Species Using HPLC ICP MS," Fresenius Journal of Analytical Chemistry, Vol. 364, No. 5, 1999, pp. 462-466. doi:10.1007/s002160051368

[157] I. Martin, M. A. Lopezgonzalvez, M. Gomez, C. Camara and M. A. Palacios, "Evaluation of High-Performance Liquid-Chromatography for the Separation and Determination of Arsenic Species by Online HPLC HG AAS," Journal of Chromatography B-Biomedical Applications, Vol. 666, No. 1, 1995, pp. 101-109. doi:10.1016/0378-4347(94)00567-O

[158] C. Shen-Tu, Y. C. Fan, Y. Z. Hou, K. X. Wang and Y. Zhu, "Arsenic Species Analysis by Ion ChromatographyBianode Electrochemical HG AFS," Journal of Chromatography A, Vol. 1213, No. 1, 2008, pp. 56-61. doi:10.1016/j.chroma.2008.10.016

[159] D. Heitkemper, J. Creed and J. Caruso, "Speciation of Arsenic in Urine Using HPLC with ICP MS Detection," Journal of Analytical Atomic Spectrometry, Vol. 4, 1989, pp. 279-284. doi:10.1039/ja9890400279

[160] L. S. Milstein, A. Essader, E. D. Pellizzari, R. A. Fer- 
nando and O. Akinbo, "Selection of a Suitable Mobile Phase for the Speciation of Four Arsenic Compounds in Drinking Water Samples Using Ion-Exchange Chromatography Coupled to ICP MS," Environment International, Vol. 28, No. 4, 2002, pp. 277-283. doi:10.1016/S0160-4120(02)00035-1

[161] Z. L. Chen, K. F. Akter, M. Mahmudur and R. R. Naidu, "Speciation of Arsenic by Ion Chromatography ICP MS Using Ammonium Eluents," Journal of Separation Science, Vol. 29, 2006, pp. 2671-2676. doi:10.1002/jssc. 200500304

[162] J. Zheng, H. Hintelmann, B. Dimock and M. S. Dzurko, "Speciation of Arsenic in Water, Sediment, and Plants of the Moira Watershed, Canada, Using HPLC Coupled to High Resolution ICP MS," Analytical and Bioanalytical Chemistry, Vol. 377, No. 1, 2003, pp. 14-24. doi:10.1007/s00216-003-1920-3

[163] J. Zheng and H. Hintelmann, "Hyphenation of High Performance Liquid Chromatography with Sector Field ICP MS for the Determination of Ultra-Trace Level Anionic and Cationic Arsenic Compounds in Freshwater Fish," Journal of Analytical Atomic Spectrometry, Vol. 19, No. 1, 2004, pp. 191-195. doi:10.1039/b304890j

[164] T. Lindemann, M. Hamester, J. Hinrichs and J. Wills, "High Sensitivity Arsenic Speciation: HPLC Sector Field ICP MS," Application Note Thermo, AN30012_E, 2003.

[165] L. Ebdon, S. Fitzpatrick and M. E. Foulkes, "The Speciation of Arsenic Compounds," Chemia Analityczna, Vol. 47, No. 2, 2002, pp. 179-188.

[166] A. Woller, H. Garraud, J. Boisson, A. M. Dorthe, P. Fodor and O. F. X. Donard, "Simultaneous Speciation of Redox Species of Arsenic and Selenium Using an Anion-Exchange Microbore Column Coupled with a Micro-Concentric Nebulizer and an ICP MS as Detector," Journal of Analytical Atomic Spectrometry, Vol. 13, No. 2, 1998, pp. 141-149. doi:10.1039/a704772j

[167] E. H. Larsen and S. Sturup, "Carbon-Enhanced ICP MS Detection of Arsenic and Selenium and Its Application to Arsenic Speciation," Journal of Analytical Atomic Spectrometry, Vol. 9, No. 10, 1994, pp. 1099-1105. doi:10.1039/ja9940901099

[168] M. Pettine, B. Casentini, D. Mastroianni and S. Capri, "Dissolved Inorganic Carbon Effect in the Determination of Arsenic and Chromium in Mineral Waters by ICP MS," Analytica Chimica Acta, Vol. 599, No. 2, 2007, pp. 191-198. doi:10.1016/j.aca.2007.08.016

[169] Y. Martinez-Bravo, A. F. Roig-Navarro, F. J. Lopez and F. Hernandez, "Multielemental Determination of Arsenic, Selenium and Chromium (VI) Species in Water by HPLC ICP MS," Journal of Chromatography A, Vol. 926, No. 2, 2001, pp. 265-274. doi:10.1016/S0021-9673(01)01062-7

[170] Q. L. Xie, R. Kerrich, E. Irving, K. Liber and F. AbouShakra, "Determination of Five Arsenic Species in Aqueous Samples by HPLC Coupled with a Hexapole Collision Cell ICP MS," Journal of Analytical Atomic Spectrometry, Vol. 17, No. 9, 2002, pp. 1037-1041. doi:10.1039/b202172b
[171] A. Castillo, A. F. Roig-Navarro and O. J. Pozo, "Capabilities of Microbore Columns Coupled to ICP MS in Speciation of Arsenic and Selenium," Journal of Chromatography A, Vol. 1202, No. 2, 2008, pp. 132-137. doi:10.1016/j.chroma.2008.06.031

[172] X. R. Zhang, R. Cornelis, J. deKimpe and L. Mees, "Speciation of Toxicologically Important Arsenic Species in Human Serum by Liquid Chromatography Hydride Generation AAS," Journal of Analytical Atomic Spectrometry, Vol. 11, No. 11, 1996, pp. 1075-1079. doi:10.1039/ja9961101075

[173] F. M. Pan, J. F. Tyson and P. C. Uden, "Simultaneous Speciation of Arsenic and Selenium in Human Urine by HPLC ICP MS," Journal of Analytical Atomic Spectrometry, Vol. 22, No. 8, 2007, pp. 931-937. doi: $10.1039 / \mathrm{b} 703713 \mathrm{a}$

[174] R. G. Brennan, S. Murdock, M. Farmand, K. Kahen, S. Samii, J. M. Gray, et al., "Nano-HPLC ICP MS for Arsenic Speciation," Journal of Analytical Atomic Spectrometry, Vol. 22, No. 9, 2007, pp. 1199-1205. doi:10.1039/b703257a

[175] C. B'Hymer and J. A. Caruso, "Evaluation of HPLC Systems for the Separation and Quantification of Arsenic Compounds from Apple Extracts," Journal of Liquid Chromatography \& Related Technologies, Vol. 25, No. 4, 2002, pp. 639-653. doi:10.1081/JLC-120008817

[176] J. Mattusch, R. Wennrich, A. C. Schmidt and W. Reisser, "Determination of Arsenic Species in Water, Soils and Plants," Fresenius Journal of Analytical Chemistry, Vol. 366, No. 2, 2000, pp. 200-203. doi: $10.1007 / \mathrm{s} 002160050039$

[177] A. C. Schmidt, W. Reisser, J. Mattusch, R. Wennrich and K. Jung, "Analysis of Arsenic Species Accumulation by Plants and the Influence on Their Nitrogen Uptake," Journal of Analytical Atomic Spectrometry, Vol. 19, No. 1, 2004, pp. 172-177. doi:10.1039/b307410m

[178] S. Karthikeyan and S. Hirata, "Ion Chromatography ICP MS Determination of Arsenic Species in Marine Samples," Applied Organometallic Chemistry, Vol. 18, No. 7, 2004, pp. 323-330. doi:10.1002/aoc.642

[179] R. G. Wuilloud, J. C. Altamirano, P. N. Smichowski and D. T. Heitkemper, "Investigation of Arsenic Speciation in Algae of the Antarctic Region by HPLC ICP MS and HPLC-ESI-Ion Trap MS," Journal of Analytical Atomic Spectrometry, Vol. 21, No. 11, 2006, pp. 1214-1223. doi: $10.1039 / \mathrm{b} 607203 \mathrm{~h}$

[180] S. D. Conklin, P. A. Creed and J. T. Creed, "Detection and Quantification of a Thio-Arsenosugar in Marine Molluscs by IC ICP MS with an Emphasis on the Interaction of Arsenosugars with Sulfide as a Function of $\mathrm{pH}$," Journal of Analytical Atomic Spectrometry, Vol. 21, No. 9, 2006, pp. 869-875. doi:10.1039/b608845g

[181] R. Y. Wang, Y. L. Hsu, L. F. Chang and S. J. Jiang, "Speciation Analysis of Arsenic and Selenium Compounds in Environmental and Biological Samples by Ion Chromatography-Inductively Coupled Plasma Dynamic Reaction Cell Mass Spectrometer," Analytica Chimica Acta, Vol. 590, No. 2, 2007, pp. 239-244. 
doi:10.1016/j.aca.2007.03.045

[182] R. E. Paproski and X. C. Le, "Boric Acid-Assisted Anion-Exchange Chromatography for Separating Arsenic Species," Analytica Chimica Acta, Vol. 526, No. 1, 2004, pp. 69-76. doi:10.1016/j.aca.2004.09.057

[183] S. Saverwyns, X. R. Zhang, F. Vanhaecke, R. Cornelis, L. Moens and R. Dams, "Speciation of Six Arsenic Compounds Using HPLC ICP MS with Sample Introduction by Thermospray Nebulization," Journal of Analytical Atomic Spectrometry, Vol. 12, No. 9, 1997, pp. 10471052. doi:10.1039/a701554b

[184] S. McSheehy and J. Szpunar, "Speciation of Arsenic in Edible Algae by Bi-Dimensional Size-Exclusion Anion Exchange HPLC with Dual ICP MS and Electrospray MS/MS," Journal of Analytical Atomic Spectrometry, Vol. 15, 2000, pp. 79-87. doi:10.1039/a906890b

[185] M. Raessler, B. Michalke, S. Schulte-Hostede and A. Kettrup, "Long-Term Monitoring of Arsenic and Selenium Species in Contaminated Groundwaters by HPLC and HG-AAS," Science of the Total Environment, Vol.
258, No. 3, 2000, pp. 171-181.

doi:10.1016/S0048-9697(00)00535-0

[186] J. A. Day, M. Montes-Bayon, A. P. Vonderheide and J. A. Caruso, "A Study of Method Robustness for Arsenic Speciation in Drinking Water Samples by Anion Exchange HPLC ICP MS," Analytical and Bioanalytical Chemistry, Vol. 373, No. 7, 2002, pp. 664-668. doi:10.1007/s00216-002-1384-x

[187] M. Segura, J. Munoz, Y. Madrid and C. Camara, "Stability Study of As (III), As (V), MMA and DMA by Anion Exchange Chromatography and HG-AFS in Wastewater Samples," Analytical and Bioanalytical Chemistry, Vol. 374, No. 3, 2002, pp. 513-519. doi:10.1007/s00216-002-1492-7

[188] M. J. Watts, M. Button, T. S. Brewer, G. R. T. Jenkin and C. F. Harrington, "Quantitative Arsenic Speciation in Two Species of Earthworms from a Former Mine Site," Journal of Environmental Monitoring, Vol. 10, No. 6, 2008, pp. 753-759. doi:10.1039/b800567b 